\title{
Rhythms of life: circadian disruption and brain disorders across the lifespan
}

Ryan W. Logan and Colleen A. McClung*

Abstract | Many processes in the human body — including brain function — are regulated over the 24-hour cycle, and there are strong associations between disrupted circadian rhythms (for example, sleep-wake cycles) and disorders of the CNS. Brain disorders such as autism, depression and Parkinson disease typically develop at certain stages of life, and circadian rhythms are important during each stage of life for the regulation of processes that may influence the development of these disorders. Here, we describe circadian disruptions observed in various brain disorders throughout the human lifespan and highlight emerging evidence suggesting these disruptions affect the brain. Currently, much of the evidence linking brain disorders and circadian dysfunction is correlational, and so whether and what kind of causal relationships might exist are unclear. We therefore identify remaining questions that may direct future research towards a better understanding of the links between circadian disruption and CNS disorders.

Circadian rhythms are near-24-hour oscillations found in essentially every physiological process in the human brain and body ${ }^{1}$. The suprachiasmatic nucleus (SCN) in the hypothalamus serves as the master pacemaker that sets the timing of rhythms by regulating neuronal activity, body temperature and hormonal signals ${ }^{2}$ (FIG. 1).

In individual cells, molecular rhythms are generated by a transcriptional-translational feedback loop involving core transcriptional activators - circadian locomotor output cycles kaput (CLOCK), the closely related neuronal PAS domain protein 2 (NPAS2) and brain and muscle ARNT-like protein 1 (BMAL1) - that regulate the expression of many genes, including those encoding period (PER) and cryptochrome (CRY) ${ }^{1}$, which, once translated, inhibit their own transcription. Many other proteins, including various kinases, phosphatases and other transcriptional cofactors, regulate this core molecular clock (FIG. 1).

Circadian rhythms are set by both genetic and environmental factors. Most people have sleep-wake and activity rhythms that, in the absence of environmental cues, are slightly longer than 24 hours ${ }^{3,4}$, but the length of this period can be affected by circadian gene variants ${ }^{5}$. Environmental factors such as light exposure, social cues, meal times and work schedules also influence the period, phase and amplitude of these rhythms ${ }^{6,7}$.

Circadian rhythms emerge during early infancy but undergo various changes through the lifespan and with ageing (FIG. 2). In general, the timing of sleep onset and waking and other biological rhythms (for example, fluctuations in melatonin levels) is earlier relative to adults during early childhood ${ }^{8,9}$ and shifts later during adolescence, and this shift in timing may be conserved among rodents and non-human primates (NHPs) ${ }^{10}$. In older adults, rhythms often return to being substantially earlier, and this shift may be accompanied by a weakening of circadian rhythms ${ }^{11,12}$.

Circadian dysfunction is observed in several brain disorders, typically emerging at different stages of life. Disruption of circadian rhythms is associated with higher risk of brain disorders. For example, chronic shift-workers (workers with rotating schedules or consistent night shifts) are vulnerable to various diseases ${ }^{13,14}$, including psychiatric disorders such as depression ${ }^{15-19}$. Much of the currently available evidence linking brain disorders to circadian dysfunction is correlational and may not be surprising, given the importance of circadian rhythms for brain function ${ }^{20}$. Identifying causal relationships between rhythm dysfunction and psychiatric disorders may have important implications for the treatment of disorders.

In psychiatry, neurology and behavioural medicine, novel circadian-based interventions are rare and often overlooked. Even in cases in which circadian disruption is not a primary cause of a disorder, stabilizing sleepwake patterns using behavioural, environmental or pharmacological tools might, for example, ease symptoms. Understanding the extent to which circadian rhythms are involved in normal functioning and pathological processes requires systems-biology approaches. Such approaches have proved valuable for investigating the complex relationships between rhythms and metabolic disorders ${ }^{21}$ and continue to provide strong translational potential for treating metabolic disorders and 
a Circadian timing system

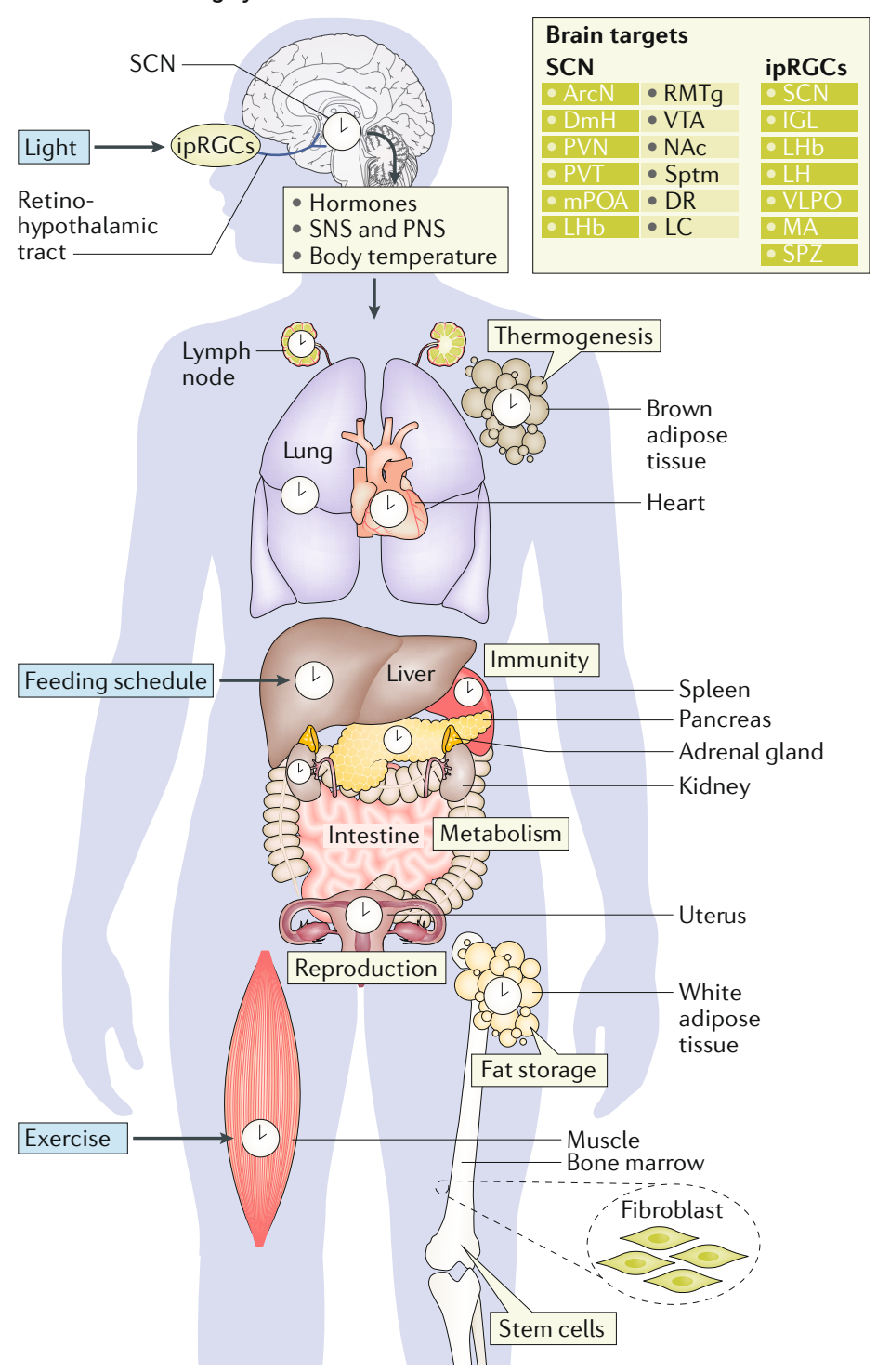

b Mammalian molecular clock
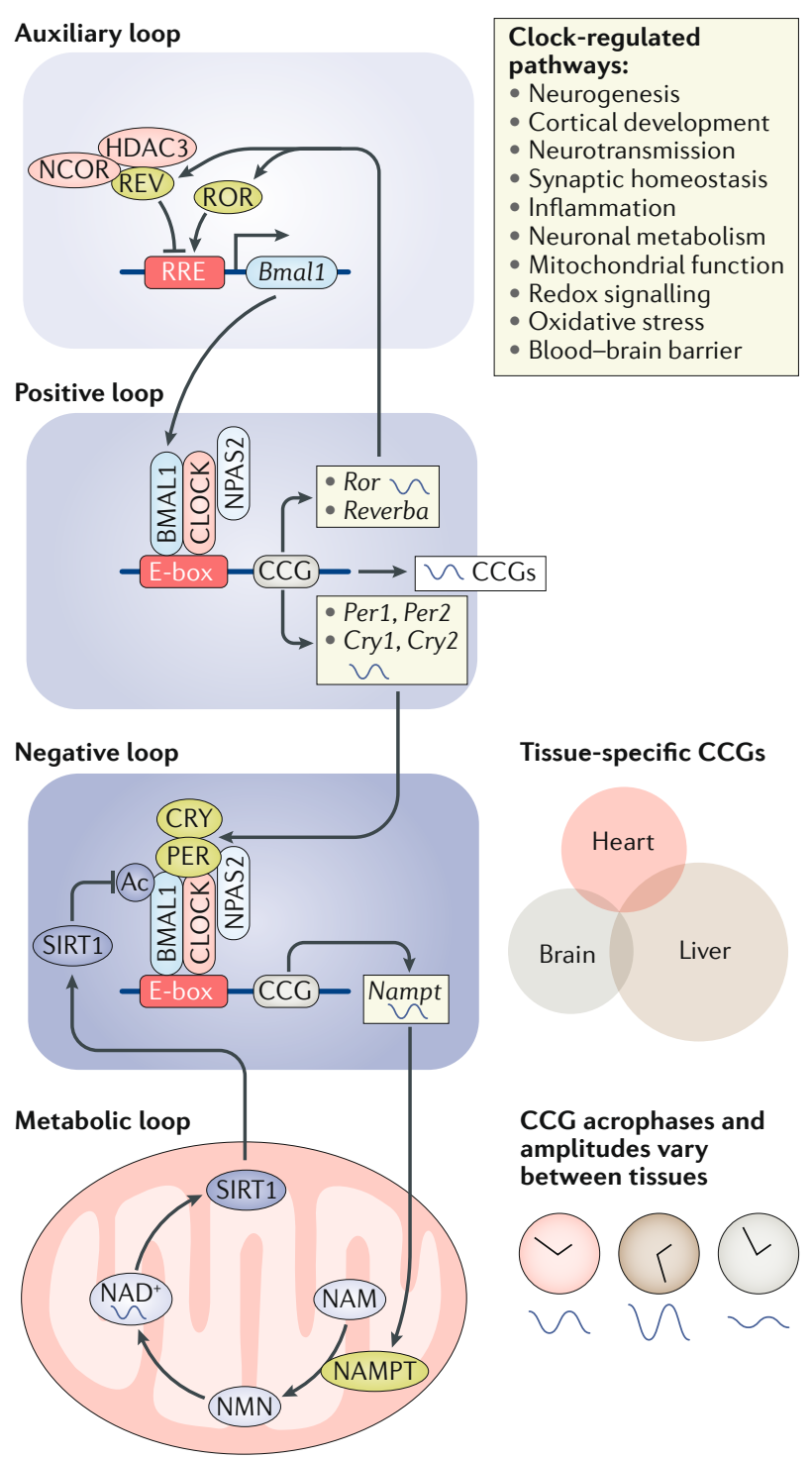

Tissue-specific CCGs

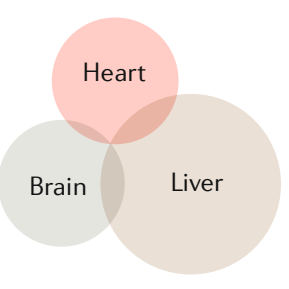

CCG acrophases and amplitudes vary between tissues

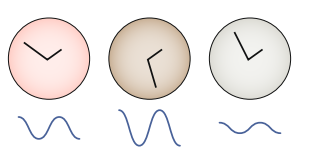

Fig. 1 | The circadian timing system. a | The circadian timing system synchronizes clocks across the entire body to adapt and optimize physiology to changes in our environment. Light is received by specialized melanopsinproducing photoreceptive retinal ganglion cells (ipRGCs) in the eye. These ipRGCs project through the retinohypothalamic tract to the suprachiasmatic nucleus (SCN), among other brain regions. The $\mathrm{SCN}$ relays timing information to other areas of the brain via direct projections (dark green boxes) and indirect projections (light green boxes). Humoral signals and the peripheral nervous system (that is, the sympathetic nervous system (SNS) and parasympathetic nervous system (PNS)) convey information from the SCN to orchestrate peripheral clocks. Feeding schedules and exercise can also entrain central and peripheral clocks. Circadian rhythms are key regulators of thermogenesis, immune function, metabolism, reproduction and stem cell development. $\mathbf{b} \mid$ The mammalian molecular clock is composed of transcriptional and translational feedback loops that oscillate with a near-24-hour cycle. The positive loop is driven by the heterodimerization of either circadian locomotor output cycles protein kaput (CLOCK) or neuronal PAS domain-containing protein 2 (NPAS2) with brain and muscle ARNT-like 1 (BMAL1) in the nucleus. The resulting heterodimers bind to enhancer boxes (E-boxes) in gene promoters to regulate the transcription of clockcontrolled genes (CCGs), including those encoding period (PER) proteins and cryptochrome (CRY) proteins. PER and CRY proteins accumulate in the cytoplasm during the circadian cycle, eventually dimerizing and shuttling to the nucleus to inhibit their own transcription, thus closing the negative-feedback loop. The auxiliary loop includes the nuclear retinoic acid receptor-related orphan receptors (RORa and ROR $\beta$ ) and REV-ERBs (REV-ERB $\alpha$ and REV-ERB $\beta$ ), which are also transcriptionally regulated by CLOCK-BMAL1 heterodimers. REV-ERBa (REV in the figure) and ROR $\alpha$ repress and activate the transcription of Bmal1, respectively, by inhibiting and activating the ROR or REV-ERB response elements (RREs). CLOCKBMAL1 complexes also control the expression of nicotinamide phosphoribosyltransferase (NAMPT), which is the rate-limiting enzyme of NAD+ biosynthesis from nicotinamide (NAM). NAM is modified by NAMPT to produce nicotinamide mononucleotide (NMN), which in turn is converted to $\mathrm{NAD}^{+}$by several adenyltransferases. Thus, NAMPT oscillations control circadian fluctuations in NAD levels, which in turn modulate sirtuin 1 (SIRT1) activity and signalling. High levels of $\mathrm{NAD}^{+}$promote SIRT1 activation. SIRT1 interacts directly with CLOCK-BMAL1 to deacetylate BMAL1 and inhibit CLOCK-driven transcription. Between tissues and cell types, CCGs and other molecular and cellular rhythms may be expressed with different acrophases (phase of peak expression), amplitudes and even periodicities. ArcN, arcuate nucleus; DmH, dorsomedial hypothalamus; DR, dorsal raphe; IGL, intergeniculate leaflet; LC, locus coeruleus; LH, lateral hypothalamus; $\mathrm{LHb}$, lateral habenula; MA, medial amygdala; $\mathrm{mPOA}$, medial preoptic area; NAc, nucleus accumbens; PVN, paraventricular nucleus of the hypothalamus; PVT, paraventricular nucleus of the thalamus; RMTg, rostromedial tegmental nucleus; Sptm, septum; SPZ, subparaventricular zone; VLPO, ventrolateral preoptic nucleus; VTA, ventral tegmental area. 

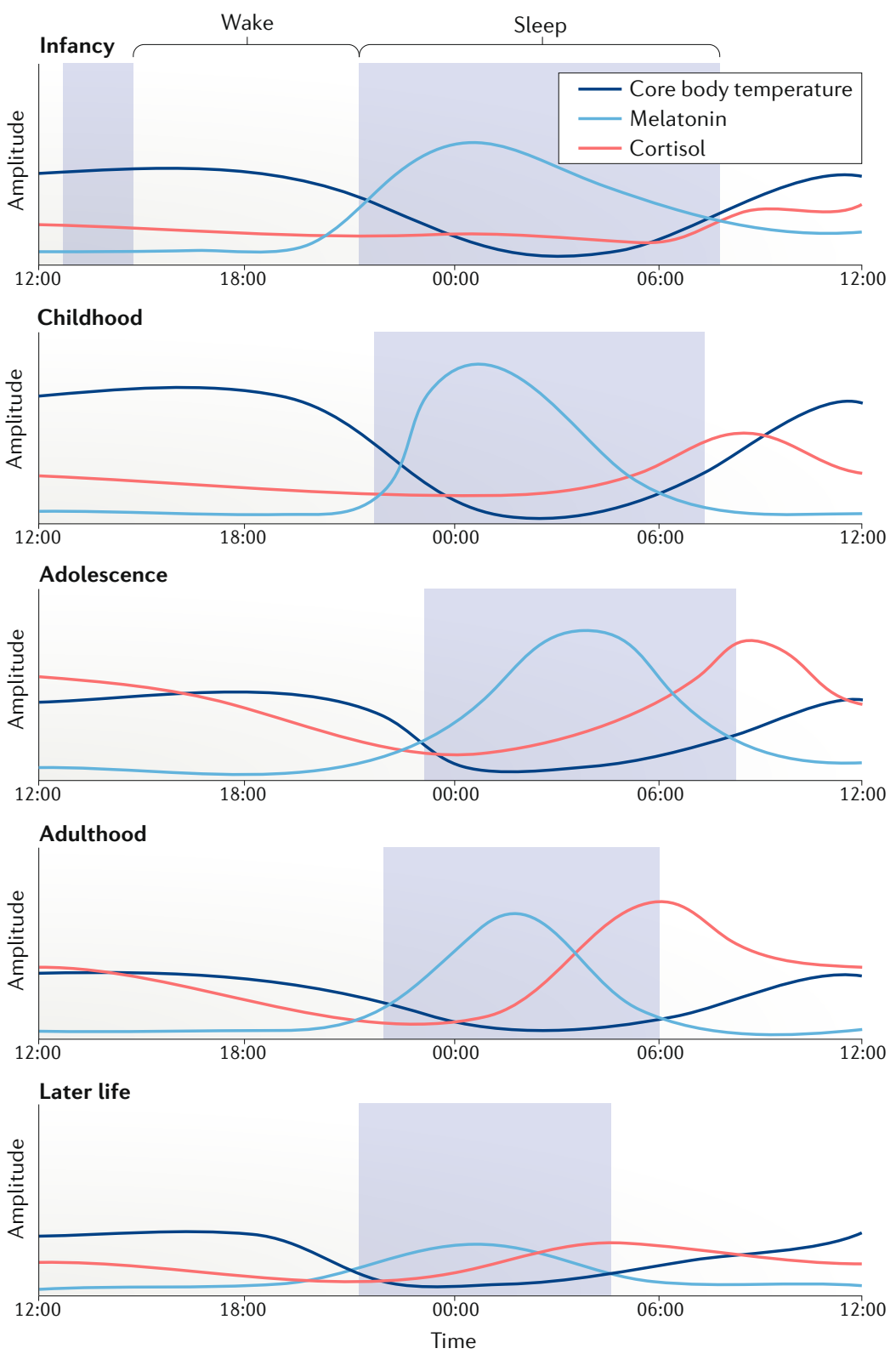

Fig. 2 | Rhythms across the lifespan. Schematic of circadian rhythm changes from infancy, adolescence, adulthood and older age. During infancy, sleep-wake rhythms are ultradian and consolidate during the first year of development. From childhood to adolescence, there is a marked shift from an early to a late chronotype, which subsequently becomes earlier during adulthood, with shorter sleep durations through adulthood. Rhythms undergo a gradual loss of amplitude with ageing. Temperature rhythms peak during childhood, and amplitudes steadily reduce during ageing. Melatonin rhythms are delayed during adolescence, with overall levels peaking during childhood and considerably decreasing during ageing. Similarly, rodent studies have demonstrated that suprachiasmatic nucleus (SCN) activity rhythms gradually decline with ageing (not shown). Cortisol rhythms peak earlier in the morning during childhood and, with age, gradually widen and reduce in overall amplitude. The amplitude of rhythmic gene expression in the brain and other tissues is reduced during ageing, affecting tissue homeostasis and function (not shown).

premature ageing through molecular and behavioural therapeutics targeting the circadian system ${ }^{22}$. In this Review, we describe the changes in circadian rhythms that occur with human development into adulthood and with ageing and highlight evidence suggesting that circadian disruptions at different life stages may be associated with certain brain disorders that typically emerge during these life periods, such as neurodevelopmental, psychiatric or neurodegenerative disorders. As most of the research linking circadian disruption and brain disorders is correlational, we emphasize key findings with a particular focus on mechanism and causality. In addition, we discuss implications for treatments and interventions.

\section{Prenatal period and early childhood}

Many of our insights into the development of the circadian system are from laboratory investigations of rodents and NHPs. By mid-to-late gestation, rhythms of metabolic activity and gene expression are evident in the SCN of rodents and $\mathrm{NHPs}^{23-25}$; however, the functional importance of early prenatal development of the SCN for fetal rhythms and physiology is less well understood. In humans, melatonin and dopamine receptors appear as early as gestational week 18 in the fetal $\mathrm{SCN}^{26-28}$, suggesting melatonin and dopamine may serve as the primary communicators of circadian information for the fetus $\mathrm{s}^{29,30}$. Melatonin from the mother readily passes through the placenta and the fetal blood-brain barrier and may thus be the predominant relay of time-of-day information to the fetus, particularly during the transition from dusk to night, as circulating melatonin rises ${ }^{31-34}$. Circadian rhythms of melatonin along with corticosteroid are detectable in the umbilical artery and vein, along with fetal circulation ${ }^{35,36}$. Fetal rhythms are also entrained by maternal core body temperature, feeding and hormone release ${ }^{27}$ (FIG. 3).

Prenatal and maternal rhythms. Epidemiological studies report increased risks of preterm delivery, low birthweights and miscarriage in pregnant women who are chronic shift-workers ${ }^{37-40}$. Abnormal sleep, feeding and work schedules might disrupt maternal rhythms, such as melatonin fluctuations, and thus desynchronize the maternal SCN from peripheral oscillators and lead to deleterious effects of shift-work on the fetus. Pregnant rats exposed to simulated shift-work (that is, complete reversal of the light-dark cycle every 3-4 days for several weeks) showed substantially reduced weight gain during early pregnancy and reduced fat-pad and liver weights, and also exhibited lower-amplitude rhythms of corticosterone, glucose, insulin and leptin levels ${ }^{41}$. The same manipulation also affected metabolic and circadian gene expression rhythms in the fetal liver, but did not affect placental or fetal growth ${ }^{41}$. Exposing pregnant NHPs to constant light suppresses the emergence of melatonin and body temperature rhythms in their offspring following birth ${ }^{42-44}$, suggesting that maternal rhythms are important for the early brain development of fetal circadian systems ${ }^{45}$. Moreover, the rhythms of the expression of circadian genes (specifically, Bmall (also known as Arntl) and Per2) and genes encoding NMDA receptor subunits (including Grin1b, Grin $3 a$ and Grin $3 b$ ) are suppressed in the hippocampus of adult offspring of rats exposed to constant light during pregnancy ${ }^{46}$. Alterations in the rhythms of circadian gene expression are also associated with impaired spatial memory in these offspring ${ }^{46,47}$, and these memory deficits can be 

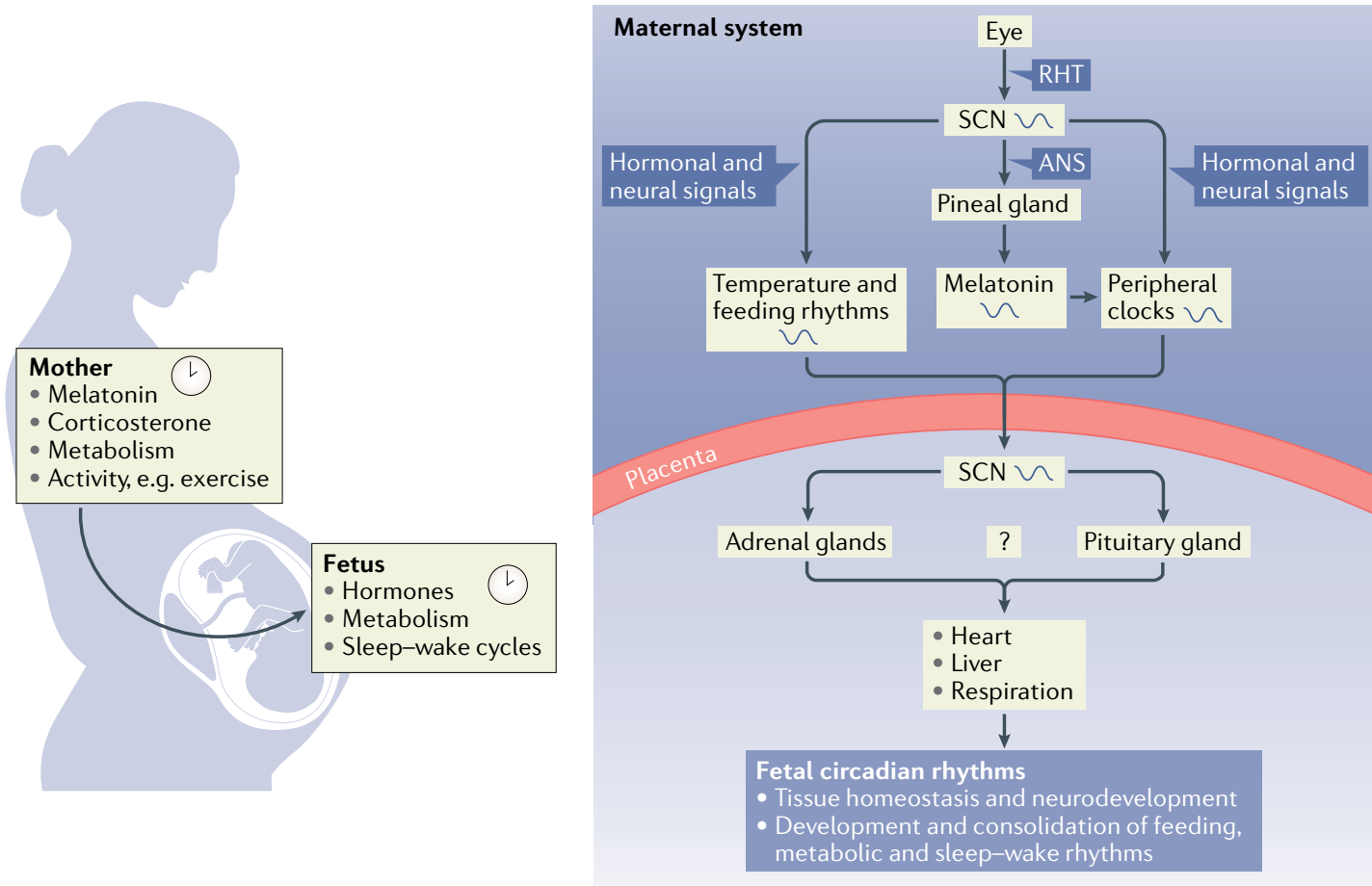

Fig. 3 | Maternal and fetal rhythms. Fetal tissues such as adrenal glands and the suprachiasmatic nucleus (SCN) may be entrained by rhythms of maternal feeding schedules, core body temperature and melatonin. Entraining signals, including melatonin and glucocorticoids, may cross the placental barrier to entrain, modulate or aid the development of fetal circadian rhythms among brain and peripheral tissues. ANS, autonomic nervous system; RHT, retinohypothalamic tract.

\section{Peripheral oscillators}

Circadian 'clocks' in other parts of the brain and in peripheral organs that are driven by the suprachiasmatic nucleus and various other nonphotic cues and stimuli.

\section{Actigraphy}

The monitoring of physical activity patterns (that is, restactivity cycles) over many days, weeks or months, typically using non-invasive devices, such as wrist actometers.

Chronotype

The preference for early

or late activities (translating

to morning or evening

chronotypes, respectively)

during the 24-hour cycle.

Sleep homeostasis

A type of homeostasis by

which prolonged periods of wakefulness lead to increases

in the intensity and duration of subsequent sleep, in

compensation for a sleep deficit or deprivation. prevented by regularly scheduled supplementation of melatonin to the mother ${ }^{46}$. Gestational circadian disruption also promotes social avoidance and hyperactivity behaviours in pups, even when they are cross-fostered by non-disrupted mothers ${ }^{48}$, further highlighting the importance of circadian stability during pregnancy on the mother and offspring.

Other studies have shown that even dim light at night has physiological and behavioural consequences on parents and offspring. For example, chronically exposing mice to dim light at night impairs adaptive immune function $^{49}$ and increases depression-like behaviours ${ }^{50}$ in their offspring. Notably, these effects are evident in offspring from parents exposed to dim light at night before breeding, suggesting that repeated exposure to dim light at night leads to epigenetic modifications that can be passed between generations. Such studies are particularly important because rates of shift-work and extended work schedules have increased over the past decade, as have exposures to prolonged periods of light at night from work and social demands, and the use of electronic devices ${ }^{51}$.

Rhythms in early childhood. Circadian rhythms gradually emerge between birth and the first several months of life. Temperature rhythms appear almost immediately after birth in full-term infants, whereas other rhythms, including rest-activity, sleep-wake and hormonal cycles, typically develop between 3 and 6 months of age $\mathrm{e}^{23,52}$. During the first year of life, sleepwake rhythms continue to consolidate, coinciding with increased melatonin secretion at sunset ${ }^{23}$. Notably, nocturnal sleep onset is coupled with sunset for the first few months after birth and then to family bedtime thereafter, suggesting that the circadian system is initially entrained by light but is subsequently entrained by social and environmental factors. Most infants, toddlers (2-3-year-olds) and young children (4-11-yearolds) display earlier sleep-wake patterns and a stronger morning preference than do adults, according to the Children's Chronotype Questionnaire - even when considering the high intra-individual and interindividual variability of sleep onset, mid-points and wake times measured by parental report questionnaires and actigraphy $^{8,9}$. However, on non-scheduled days, children tend to wake up later, substantially delaying their sleepwake times, indicating that social and environmental factors, such as schedules imposed by school and parents, have a major influence on survey-based measures of chronotype ${ }^{9}$. Importantly, children who consistently display earlier sleep onsets on scheduled days tend to delay less than do children who have later sleep onsets and wake times ${ }^{9}$.

Social environment and scheduling demands by the parents can interact with endogenous circadian and sleep rhythms, creating a state of misalignment between social schedule, endogenous circadian physiology and sleep homeostasis. Sleep loss and consistently shorter sleep durations have been linked to inattentiveness, frustration and hyperactivity in 2-5-year-old children ${ }^{53,54}$. Interestingly, before the age of 3 years, poor sleep quality and shorter sleep durations, which could be the result of inconsistent sleep-wake cycles and less-robust circadian rhythms, are predictive of hyperactivity, cognitive 
Melanopsin photoreceptors Opsin class G protein-coupled receptors expressed in a small percentage ( $\sim 2 \%$ ) of photosensitive mammalian retinal ganglion cells.

Bright light therapy A therapy for mood and sleep disorders that involves the use of bright light, typically in the morning, to shift and stabilize endogenous circadian timing.

Sleep efficiency

A measure of the integrity of

sleep architecture and quality of sleep, consisting of the ratio of total sleep time to the amount of time spent in bed.

\section{Narcolepsy}

A chronic neurological disorder characterized by excessive daytime sleepiness, with many individuals experiencing intermittent, uncontrollable episodes of cataplexy (a sudden loss of muscle tone during wakefulness)

Amphetamine

A potent stimulant drug that can be used in the treatment of attention-deficit hyperactivity disorder

Methylphenidate

A stimulant drug primarily used in the treatment of attention-deficit hyperactivity disorder. deficits and impulsivity at 6 years of age ${ }^{55,56}$, even in children showing normative sleep in the interim period ${ }^{57}$. Inconsistent sleep-wake cycles during early development might be contributing to the steadily rising rates of emotional and behavioural problems in young children $^{58-61}$. Additional - ideally longitudinal - studies from infancy and through early childhood are needed to better understand the interplay between environment and biology in the development of the circadian system and the rest of the brain and their potential involvement in cognitive, mood and behavioural issues. In support of this, a large twin study suggested that shared environments (rather than genetics) are the primary influencer of sleep abnormalities and behavioural problems ${ }^{62}$, although these findings do not preclude the involvement of genetic or other biological factors that shape early parenting practices.

At birth, light is the most important stimulus for entraining circadian rhythms. Rodent studies demonstrate that melanopsin photoreceptors are present prenatally, are light-responsive after gestation and undergo functional reorganization and maturation during the first few weeks of life ${ }^{63-69}$. In rats, photic inputs to the SCN are mature after postnatal day $10\left(\mathrm{REF}^{64}\right)$, whereas in mice, these pathways reach maturity by postnatal day 14 , when the eyes fully open ${ }^{67,69}$. A few studies have shown that the eyes of infants who were born prematurely can respond to light as early as gestational week 30 , suggesting that photic input pathways may be functional during the third trimester of development ${ }^{70,71}$. Studies in baboons reveal that metabolic activity and gene expression in the SCN are highly responsive to light inputs in full-term infants and can be entrained by low-intensity light, similar to human infants $\mathrm{s}^{72-74}$.

Regular light-dark schedules and/or low-intensity lighting, as well as particular wave lengths of light that affect the circadian clock, may be beneficial for the overall health of premature infants $\mathrm{s}^{75-77}$. In neonatal intensive care units (NICUs) worldwide, constant lighting conditions are often used to aid the care and nursing staff to readily respond to potential emergencies and frequent health monitoring ${ }^{78}$. However, rearing premature infants (32 weeks gestational age or older) under regular lightdark schedules in the NICU leads to greater and more rapid weight gain than rearing infants under constant bright light or dim light, effectively shortening their hospital stays ${ }^{76,77}$. Premature infants kept in regular lightdark conditions also fed orally sooner and spent fewer days on ventilator assistance, but had similar motor coordination compared with infants under continuous near darkness in the NICU (28-32 weeks of gestation $)^{79-85}$. Moreover, compared with infants in constant dim light, these infants cry less and are more active in the daytime ${ }^{81}$. Regular light-dark schedules also support the maturation of rest-activity, sleep-wake and melatonin rhythms earlier in premature infants ${ }^{71,85}$. Although more controlled comparative studies are necessary, these findings strongly suggest that regular light-dark and feeding schedules may substitute, in part, for the early loss of the influence of maternal rhythms and loss of the constant darkness environment in utero in premature infants. Larger multisite randomized trials will be necessary to determine the extent to which different light-dark schedules might improve long-term health outcomes in infants in the NICU.

\section{Neurodevelopmental disorders}

Sleep disturbances and circadian disruptions are associated with several neurodevelopmental disorders, including attention-deficit hyperactivity disorder (ADHD), autism spectrum disorders (ASDs), Prader-Willi syndrome (PWS) and Smith-Magenis syndrome (SMS). One of the most common neurodevelopmental disorders of childhood, ADHD, is characterized by inattentiveness, impulsivity and hyperactivity and is associated with high rates of co-occurring sleep problems and circadian alterations $^{86}$. Reductions in sleep quality, delays in circadian phase and evening preference are consistently reported in children and adults with $\mathrm{ADHD}^{87}$ and may be correlated with the severity of ADHD symptoms ${ }^{88}$. In adults with ADHD, a loss of circadian gene expression rhythms in the oral mucosa accompanies delays in cortisol rhythm and reduced-amplitude melatonin rhythms ${ }^{89}$. Advancing melatonin rhythms using bright light therapy improves ADHD symptoms of hyperactivity and impulsivity in adults independent of changes in sleep time, sleep efficiency, wake time or waking during sleeping ${ }^{87,90}$.

Abnormal melatonin rhythms (for example, with phase delays and reduced amplitude) are also reported in children with $\mathrm{ADHD}^{87}$. Studies of sleep-wake cycles in children with ADHD are often complicated by the effects of stimulants used to treat the disorder ${ }^{91}$. Interestingly, modafinil, a stimulant used to treat narcolepsy and shift work disorders, may be effective for treating ADHD ${ }^{92}$ and, along with targeting dopaminergic signalling, may also target therapeutic mechanisms distinct from those targeted by amphetamine and methylphenidate, including central histamine and orexin signalling pathways, to improve sleep efficiency and quality ${ }^{93}$.

Studies have also tenuously associated dysfunction in circadian rhythms with ASD, a neurodevelopmental disorder characterized by impairments in social communication, restricted interests and repetitive behaviours. Sleep problems are very common in children with $\mathrm{ASD}^{94-96}$, although revealing any underlying circadian abnormalities has proved difficult ${ }^{97,98}$. The most consistent related finding in prepubertal children, pubertal adolescents and young adults with ASD is reduced melatonin levels in the evening ${ }^{99-102}$. A genetic basis for impaired melatonin synthesis in ASD was proposed following the finding that unaffected parents of children with ASD have lower evening melatonin levels and lower activity of the acetylserotonin methyltransferase (ASMT) enzyme ${ }^{102}$, which converts $N$-acetylserotonin to melatonin ${ }^{103}$. Variants identified in the promoter of the ASMT gene were specific to individuals with ASD compared with controls and were associated with reduced ASMT activity and melatonin levels ${ }^{102}$. In addition to its role in sleep and circadian rhythms, melatonin is also a potent antioxidant. Reductions in melatonin during early development have been hypothesized to lead to a build-up of oxidative stress, which is harmful to the developing nervous system and increases the risk of neurodevelopmental disorders such as $\mathrm{ASD}^{104}$. 
A double-blind placebo-controlled study of 125 children and adolescents (2-17.5 years of age) supports the use of melatonin supplementation to treat sleep disturbances associated with $\mathrm{ASD}^{105}$ and may lead to other therapeutic approaches targeting circadian rhythms and sleep issues, as well as other symptoms of ASD ${ }^{106}$.

Other neurodevelopmental disorders, such as PWS and SMS, are linked more directly to dysfunctional circadian rhythm pathways ${ }^{107,108}$. PWS is characterized by hypotonia and failure to thrive in infancy and early toddlerhood followed by hyperphagia and childhood obesity, intellectual impairment, hypogonadism and obsessive-compulsive-related behaviours ${ }^{109}$, and it is due to a paternal deficiency of the 15q11-13 locus ${ }^{110}$. PWS is also accompanied by shorter sleep duration and excessive daytime sleepiness ${ }^{71,111,112}$. The 15q11-13 locus contains several clusters of genes encoding small nucleolar RNAs (snoRNAs), including SNORD116, which is expressed in the brain of mice and humans ${ }^{113-115}$. In mice, paternal deletion of Snord 116 increases the expression of several genes specifically during the light phase, including Ube $3 a$, which encodes ubiquitin ligase E3A $(\mathrm{UBE} 3 \mathrm{~A})^{116}$. UBE3A targets the circadian transcription factor BMAL1 for proteasomal degradation and thus is crucial for maintaining the pace of molecular clock timing $^{117}$. UBE3A belongs to a cluster of imprinted genes also within the 15q11-13 locus. Specifically, genomic imprinting, a rare epigenetic mechanism of gene regulation and developmental imprinting ${ }^{118}$, leads to parentof-origin-specific effects of deletion of the 15q11-13 locus. For example, SNORD116 is expressed exclusively from the paternally inherited allele ${ }^{113}$, whereas maternally inherited alleles of UBE3A are linked to PWS ${ }^{119,120}$. Maternal duplications of the 15q11-13 locus lead to increased expression of $U B E 3 A$ and epigenetic silencing of the paternal antisense transcript, UBE3A-ATS ${ }^{121-124}$. The maternal allele of $U B E 3 A$ is imprinted only in neurons and is expressed by alleles in other cell types ${ }^{120,125,126}$. In mice with maternal loss of Ube $3 a$, period lengthening of locomotor rhythms and molecular rhythms in the brain are dependent on core clock factors, including CLOCK and BMAL1 (REF. ${ }^{127}$ ). Intriguingly, activation of the epigenetically silenced paternal Ube $3 a$ allele restores neuronal circadian function ${ }^{127}$, suggesting that targeting the locus may be useful for treatment and involve molecular-clock-dependent mechanisms. Thus, duplication of the 15q11-13 locus and developmental imprinting of SNORD116 and UBE3A may provide a molecular explanation for the circadian rhythm disruptions common to patients with PWS.

SMS is a rare neurodevelopmental disorder characterized by craniofacial and skeletal anomalies, metabolic problems and obesity, along with intellectual disability and stereotypical behaviours, and is a result of a heterozygous deletion of chromosomal region 17p11.2. The deleted region contains several genes, including the gene encoding retinoic acid induced one (RAI1), the loss of which is thought to be responsible for many of the disease characteristics. Symptoms of SMS also include sleep and circadian abnormalities, with the most extreme being a complete reversal of sleep-wake patterns (that is, daytime sleep and night-time wake) $)^{128-131}$.
RAI1 is implicated in melatonin secretion, and inverted melatonin rhythms may be responsible for these sleep disturbances ${ }^{132}$. Mice with a heterozygous deletion of Rail display a shortened locomotor activity period ${ }^{133,134}$, although these mice are of mixed genetic background containing C57BL/6, which do not produce pineal melatonin. Introducing the mutation into another mouse background capable of generating endogenous melatonin may yield more pronounced effects on sleep and circadian rhythm phenotypes relevant for SMS. In human cells from patients with SMS and the mouse brain, RAI1 activates the transcription of CLOCK via direct binding to enhancer elements within intron 1 of the CLOCK gene $^{135}$, demonstrating a possible mechanism by which loss of RAI1 may result in circadian dysfunction at the molecular and cellular level. Further study is required to definitively link molecular clock disruption observed in animal models of SMS and patients with the disorder to the reversal in melatonin secretion. Circadian, sleep and behavioural disturbances are the most severe and difficult to treat for individuals with SMS. Attenuating the rise of melatonin during the morning via pharmacological antagonism of $\beta_{1}$-adrenergic receptors by acebutolol (as pineal melatonin production is stimulated by sympathetic nervous system activation of $\beta_{1}$ - and $\alpha_{1}$-receptors) has been effective for reducing daytime naps, improving concentration and reducing the frequency of tantrums in a sample of ten patients, aged $4-18$ years ${ }^{136}$. Combining $\beta_{1}$-adrenergic receptor antagonism with timed administration of exogenous melatonin greatly improves sleep (that is, leading to fewer sleep awakenings and better organized sleep stages) and also reduces disruptive behaviours during the daytime in children and adolescents with $\mathrm{SMS}^{137}$. Thus, therapies targeting the reversal of melatonin rhythms in patients with SMS may improve sleep, as well as behaviour, attention and learning ${ }^{137-139}$.

\section{Adolescence}

Humans, rodents and some NHPs undergo changes in circadian rhythms during adolescence ${ }^{140}$. At the onset of puberty, sleep-wake cycles and melatonin rhythms start to phase delay ${ }^{141}-$ a shift that is dependent on the presence of gonadal hormones, and that coincides with sexual maturity ${ }^{142,143}$. Environmental factors, such as social pressure from peers and/or decreasing parental involvement in bedtime routines, can also contribute to changes in adolescent sleep timing and duration ${ }^{140}$. Sensitivity to the phase-shifting effects of light may also play a role, which is especially important when considering the increasingly pervasive use of electronic devices at night and their influence on sleep timing, duration and quality ${ }^{144}$. Adolescents may be more sensitive to the ability of light exposure to shift rhythms at specific times of day. For example, light exposure during the night $(23: 00 \mathrm{~h}$ to $24: 00 \mathrm{~h})$ suppresses melatonin levels in adolescents, with greater suppression occurring in early adolescents (prepubertal to mid-pubertal adolescents aged 9-14 years) than in late adolescents (aged 11-15 years, in late pubertal to post-pubertal stages) ${ }^{145}$. Early-morning light exposure (between 03:00 h and 04:00 $\mathrm{h}$ ) also reduced melatonin, although no differences 
Eveningness

The preference for activity in the evening and later bed times, related to endogenous circadian phase, and akin to evening chronotype. were found between early and late adolescents ${ }^{145}$. Thus, the sensitivity of the circadian system to light exposure depends on the developmental stage and time of day, and early adolescents may be particularly vulnerable to the phase-delaying effects of light at night ${ }^{145}$. Although the amount of sleep needed per night is variable, the vast majority of US adolescents sleep less than the suggested 8-10 hours of sleep per night, according to the American Academy of Pediatrics ${ }^{146,147}$. Early school start times, which are typically more than an hour earlier in US high schools (ages 15-18 years) than in elementary schools (ages 9-10 years) or middle schools (ages 11-13 years), may contribute to these problems of shorter sleep ${ }^{10,148}$. On weekends, adolescents in the US tend to sleep later, both reverting to their natural sleep-wake cycle and possibly to recover from cumulative sleep loss during the school week ${ }^{149}$. Transitions from weekday to weekend compound the phase delay in the endogenous clock ${ }^{150,151}$ that is often referred to as 'social jet-lag' ${ }^{148,152}$.

Substance use disorders. Emerging evidence from studies in humans and animal models suggests that disruptions to sleep and circadian rhythms during adolescence have consequences on brain development and might contribute to the vulnerability to mood and substance use disorders (FIG. 4). Experimentation with substances often begins during mid-adolescence to late adolescence (ages $~ 14-18$ years), and the frequency of use peaks during early to mid-20s ${ }^{153}$. Circadian disruptions (such as social jet-lag) during adolescence increase vulnerability to substance use, and conversely, chronic exposure to these substances can lead to long-lasting changes in the circadian and sleep networks ${ }^{154}$. Weekday-weekend differences in sleep timing and duration are associated with increased risk-taking behaviours, substance use and depressed mood ${ }^{155,156}$. Moreover, adolescents with shorter sleep duration are more likely to use substances, including caffeine, nicotine, alcohol and marijuana ${ }^{157-159}$, and to engage in other risky behaviours ${ }^{157,160,161}$. Longitudinal follow-up studies have found that weekend delay and sleep-timing variability during adolescence and young adulthood positively correlate with alcohol use 2 years later ${ }^{162}$ and the incidence of alcohol use disorder symptoms 3 and 5 years later ${ }^{163}$ and are associated with an earlier onset of alcohol use disorder $^{164}$. Greater eveningness and later sleep timing in adolescents and young adults are associated with increased substance abuse and may not merely be a consequence of increased opportunity to engage in substance use later at night $^{165-167}$. However, the roles of biological factors versus

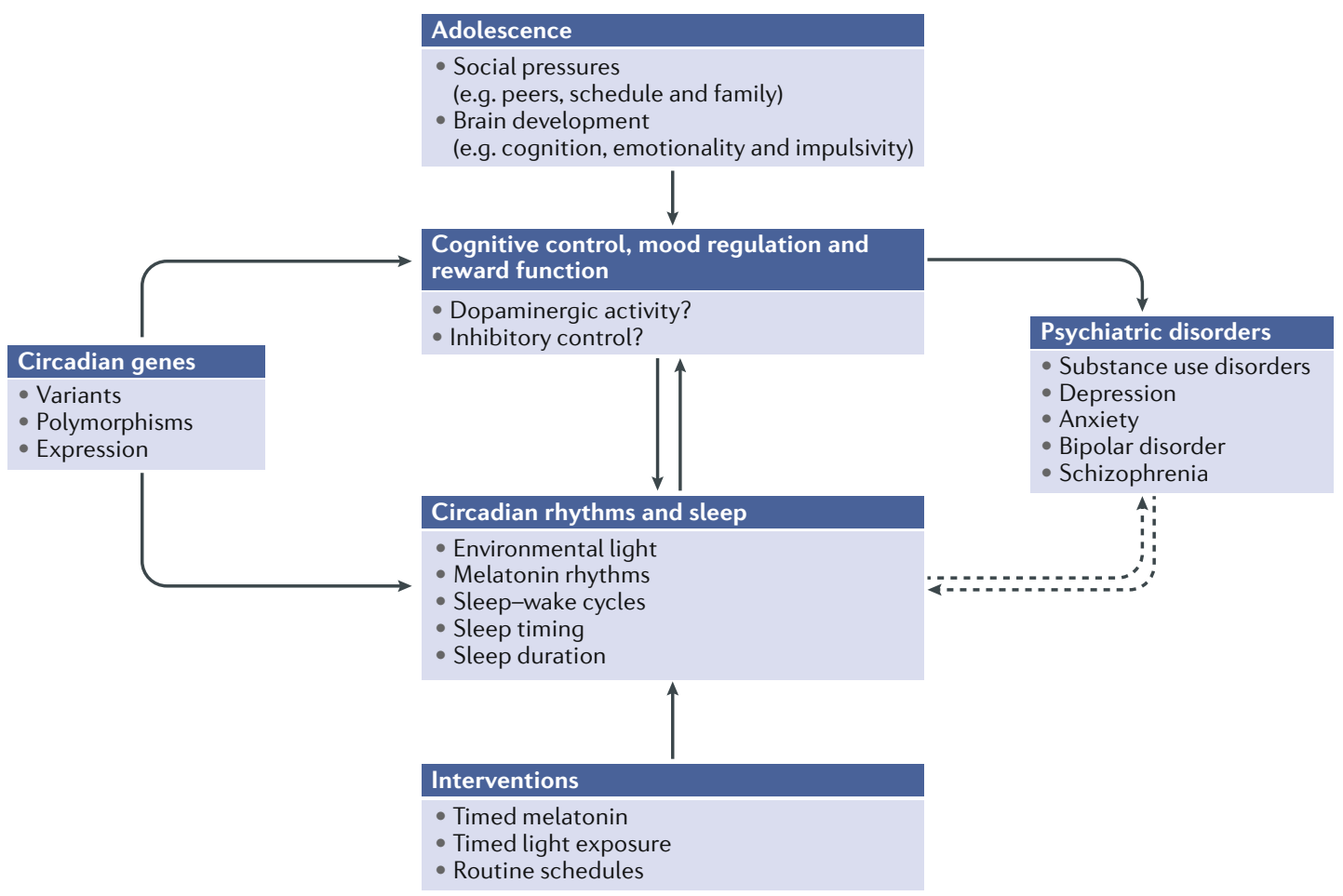

Fig. 4 | Effects of social constraints, sleep and circadian disruptions on adolescent brain function. A proposed model for the associations between sleep, circadian rhythms and psychiatric disorders during adolescence. Neural circuits responsible for controlling cognition, mood and reward mature rapidly during adolescence and may be negatively affected by sleep deprivation and circadian misalignment, potentially leading to poor decision making, impulsivity and risky behaviours. Basic and clinical studies have linked circadian genes and their variants to corticostriatal dopamine and glutamatergic signalling that are important for cognition, mood and reward. These changes contribute to the vulnerability of developing psychiatric disorders. Several psychiatric disorders, including mood disorders, substance use disorders and schizophrenia, are associated with alterations in rhythms and/or sleep that affect cognition, motivation and impulsivity. Sleep and/or circadian rhythm disruptions (for example, genetic and/or environmental perturbations) are also associated with vulnerability to and the progression of psychiatric disorders. Interventions for targeting sleep and/or circadian rhythms may be therapeutically effective for treating disorders that emerge during vulnerable periods of adolescence. 
social influences remain unclear: social context is also important, and social networks may affect sleep duration and substance use in adolescents ${ }^{168}$.

During adolescence, many developmental changes occur in the frontostriatal reward circuitry, which includes the ventral striatum (VS; including the nucleus accumbens), dorsal striatum (DS) and medial prefrontal cortex $(\mathrm{mPFC})^{169,170}$. These changes include increases in synaptic pruning and myelination ${ }^{107,171}$, as well as increases in the availability of dopamine in the limbic circuitry ${ }^{172,173}$. The combination of heightened activity in rewardrelated circuits and underdeveloped cognitive control centres contributes to greater emotionality, impulsivity and reward-seeking behaviour ${ }^{174,175}$. Circadian and sleep disruptions may amplify these responses by further reducing cortical control and increasing the activity of reward circuitry in human adolescents. Chronic exposure to drugs of abuse can lead to epigenetic changes affecting the molecular clock that may then alter function of the reward circuitry for long periods of time, creating more vulnerability for future drug abuse ${ }^{176}$.

Functional MRI studies reveal that the activation (that is, glucose uptake) of striatal and prefrontal brain regions during monetary reward tasks is greater in the afternoon or evening than in the morning in late adolescents and young adults (aged 19-24 years) ${ }^{177}$, and is related to morningness and eveningness chronotypes in adults $^{178}$. In an age-matched cohort of late adolescents, greater VS responses to reward outcome and reduced mPFC responses to reward anticipation are associated with eveningness ${ }^{179}$ and may predict alcohol dependence $^{167}$, further suggesting that certain circadian phenotypes emerging during early to late adolescence could contribute to vulnerability to substance use. In fact, larger weekday-weekend advances of sleep onset (for example, similar to the transition from early school start times to weekend schedules) are associated with lower $\mathrm{mPFC}$ reactivity to the anticipation and receipt of monetary reward in early adolescents (aged 11-13 years), even after adjusting for puberty, sex and total sleep time $\mathrm{e}^{149}$. Lower responsivity of the PFC could lead to impaired inhibition of striatal circuits and related behaviours, including impulsivity and risk-taking. Whether these relationships between neural responses to reward and alcohol use are attributable to circadian misalignment or other factors, some of which may affect chronotype, remains unclear. For example, sleep deprivation can also alter neural reward circuitry: a single night of total sleep deprivation leads to decreases in mPFC activity and increases in VS activity in response to rewarding stimuli in young adults (aged 18-25 years) ${ }^{180}$. Further investigations in humans and animals are necessary to determine how circadian misalignment and/or disruption, along with sleep deprivation, independently and together, may affect the development of reward and cognitive neural systems and whether interventions that target these systems are clinically beneficial. A recent meta-analysis performed on nine studies investigated the impact of insomnia treatment on alcohol use disorder $^{181}$. Behavioural (but not pharmacological) interventions for insomnia improved sleep quality and reduced symptoms of depression; however, there was no evidence that these interventions improved rates of alcohol abstinence, suggesting that improving sleep quality alone is not sufficient to prevent relapse. Determining whether chronobiological interventions can reduce the risk of developing substance use disorders or treat individuals with these disorders is an important and translationally relevant avenue of future research.

Mood disorders. Adolescence is a time during which serious psychiatric disorders, including major depression, bipolar disorder and schizophrenia, tend to emerge. The overall prevalence of disorders with severe impairment and/or distress during this developmental period is $22.2 \%{ }^{182}$, and the median age of onset for mood disorders is 13 years of age ${ }^{182}$. A major component of many mood, anxiety and psychotic disorders is a disrupted sleep-wake cycle ${ }^{183-188}$. In addition, circadian disruptions can precipitate mood and psychotic episodes in individuals who already have psychiatric disorders ${ }^{189-191}$. Mood symptoms can also occur in a seasonal pattern, as in seasonal affective disorder and in many cases of bipolar disorder ${ }^{192,193}$; symptoms may be related to the amount, duration and intensity of light varying across the seasons (that is, short versus long days) ${ }^{194}$. However, these disorders are very heterogeneous, and any association with disruptions in the sleep-wake cycle can be highly variable from individual to individual. Thus, similar to genetic studies, identifying a common circadian or sleep-related mechanism that is causal for all bipolar disorder or major depression is unlikely.

Similar to substance use disorders, delayed rhythms during adolescence and early adulthood are strongly associated with depression and the severity of mood symptoms ${ }^{195,196}$. More recent work has shown that circadian disruptions in adolescents who are at high risk of psychosis (that is, subthreshold symptoms of psychosis, social impairment and higher suspicion-paranoia) ${ }^{197}$ and individuals with mood disorders predict worse prognosis and symptoms in longitudinal studies ${ }^{198,199}$. Together, these findings suggest that circadian and sleep disruptions may have a role in the vulnerability to mood disorders and the precipitation of disorder symptoms.

Although the mechanisms underlying these associations remain to be elucidated, one possibility is that disrupted sleep and circadian rhythms affect the synaptic pruning and maturation of neural circuits during adolescence ${ }^{3,4,107,108,200}$. Many of these selective synaptic pruning and refinement processes occur during sleep, and sleep is crucial for proper circuit maturation and longterm memory formation in humans and animals ${ }^{201-204}$. In addition, slow-wave activity during sleep dramatically decreases specifically during adolescence; this reduction is suggested to result directly from synaptic refinement, further demonstrating important links between sleep and synaptic changes ${ }^{107,108}$. Excessive pruning or too little pruning during adolescence may be linked to the development of psychiatric disorders ${ }^{205,206}$; for example, 5 days of $50 \%$ sleep restriction in rats during early adolescence led to alterations in the fractions of projections from the motor cortex to other regions of the brain ${ }^{207}$. Therefore, insults during adolescence that affect sleep and circadian stability may contribute to 
the pathophysiology underlying the vulnerability and progression of these diseases later in life ${ }^{107,108}$. As an estimated $7 \%$ of adolescents meet clinical criteria for delayed sleep phase syndrome, and the majority of adolescents are reported to be sleep deprived ${ }^{208-210}$, delaying school start times should be universally considered as a potential intervention for curbing the effect of these conditions ${ }^{211}$.

Treatments to amplify, phase advance or delay circadian rhythms have been developed for potential use in psychiatric disorders in adults. These treatments include bright light therapy, acute sleep deprivation, interpersonal and social rhythm therapy and therapeutic melatonin agonists (such as agomelatine) $)^{189,212-214}$. Moreover, antidepressant and mood-stabilizing medications, such as lithium, valproic acid and selective serotonin reuptake inhibitors (SSRIs), all have dramatic effects on gene expression rhythms that may, at least in part, underlie their therapeutic efficacy ${ }^{215-217}$. Additionally, the rapid and lasting antidepressant effects of low-dose ketamine can be predicted based on measures of locomotor activity rhythms before treatment and on how well the treatment strengthens low-amplitude activity rhythms, suggesting that the therapeutic effects are directly related to the strengthening of the circadian system $^{218}$. Thus, rhythm alignment and amplification are important therapeutic approaches to helping prevent or treat psychiatric disorders. Such chronotherapy for these disorders, however, is in its infancy and needs to be evidence-based. Chronotherapies may also need to be personalized based on the particular needs of the individual; for example, subjects with depression are more responsive to light therapy combined with wake therapy and sleep-time stabilization if they are evening chronotypes and have a positive diurnal variation in mood, with the best mood occurring in the evening ${ }^{219}$.

A circadian rhythm disorder in which sleep timing is shifted

later by 2 or more hours relative to normative sleep-wake cycles and often accompanied by similar shifts in body-temperature and hormone rhythms.

Acute sleep deprivation A partial night or full night of sleep restriction. This approach can produce a short-term antidepressant effect.

Interpersonal and social rhythm therapy

A cognitive behavioural therapy often used for bipolar disorder that acts to stabilize sleep-wake and social schedules.

Dim light melatonin onset (DLMO). A marker of endogenous circadian phase that can be used for research or to determine the

appropriate timing of treatments.

Fatigue

The feeling of exhaustion and lack of energy. gastrointestinal dysfunction, sleep disorders, diabetes and depression ${ }^{227}$. A large study of 11,450 Canadian nurses found that the strongest relationship between work schedule and depression was in those who work rapidly rotating schedules and undefined rotating schedules (as opposed to slowly rotating schedules $)^{228}$. This study suggests more severe circadian disruptions are associated with a greater likelihood of developing depression. Another study of $\sim 14,000$ workers in electronics manufacturing in South Korea revealed that, compared with daytime workers, shiftworkers showed higher rates of insomnia, depression and suicidal ideation (with the strongest association with insomnia) $)^{229}$.

A survey of $\sim 4,000$ flight attendants as part of the US National Health and Nutrition Survey (NHANES) found that, compared with the general population, flight attendants are 2-5.7-fold more likely to have sleep disorders, depression, anxiety and/or fatigue ${ }^{230}$. Female flight attendants also have a higher prevalence of reproductive cancers, skin cancers and other conditions such as chronic bronchitis and heart disease ${ }^{230,231}$, probably as a consequence of carcinogens in cabin environments, including cosmic radiation and poor aircraft ventilation, but perhaps also exacerbated by night shift-work, irregular schedules and frequent crossing of time zones ${ }^{232,233}$. Taken together, many health problems during adulthood, including sleep problems, depression and anxiety, are linked to circadian disruptions that are caused by shift work or other environmental or genetic disruptions to the sleep-wake cycle.

\section{Later life}

Older people (ages $\sim 65$ years and older) generally sleep less and have poorer sleep efficiency, increased nighttime awakening, increased sleep latency and greater levels of daytime sleepiness ${ }^{234-236}$. Several studies have reported declines in melatonin levels with ageing, although others find no difference compared with younger people in a healthy population ${ }^{237,238}$. When other circadian outputs are measured, older individuals show lower-amplitude body temperature rhythms, with the minimum of the endogenous phase occurring nearly 2 hours earlier than in younger individuals ${ }^{239}$.

Importantly, measures of activity and physiological rhythms in older people can be confounded by health problems, medications and eye problems such as cataracts that can reduce light input to the central clock. In humans and rodent models, the amplitude of peripheral oscillatory rhythms dampens with age, but whether this effect is due to a loss of intrinsic core clock function, to dysfunction of SCN signalling and connections ${ }^{240,241}$ or to a deficit in entrainment to the environment is unclear $^{242}$. The number of vasopressin-expressing cells in the SCN in humans ${ }^{243,244}$ and rodents ${ }^{245,246}$ reduces with age, possibly altering SCN output. Moreover, some SCN cells become silent in older animals (as measured in vitro), and SCN neurons lose phase coherence with age $^{247,248}$. These SCN network changes result in desynchronization and a reduction in the amplitude of neuronal activity rhythms ${ }^{248,249}$ and, ultimately, physiological and behavioural rhythms ${ }^{241}$. 
In addition to $\mathrm{SCN}$ changes, gene expression rhythms in the human $\mathrm{mPFC}$ also decline with age; however, surprisingly, a separate set of genes becomes newly rhythmic in older people (aged 65 years and older) ${ }^{11}$. These rhythmic genes might be protective, as the individuals in this post-mortem study did not experience psychiatric or neurodegenerative disorders before death. There is also accumulating evidence linking disrupted sleep and circadian rhythms to neurodegenerative disorders in humans and animal model ${ }^{235,236}$.

Neurodegenerative disorders. In older adults, lessrobust circadian rhythms and more fragmented patterns of activity are risk factors for the development of dementia ${ }^{250}$. Moreover, the incidence of singlenucleotide polymorphisms (SNPs) in CLOCK and $B M A L 1$, and in BMAL1 and PER1, are associated with increased risk of Alzheimer disease (AD) and Parkinson disease (PD), respectively ${ }^{251-253}$. Compared with healthy aged people, individuals with $\mathrm{AD}$ or $\mathrm{PD}$ show considerably lower melatonin rhythm amplitudes and excessive sleepiness, as well as other sleep-wake cycle disturbances, such as later sleep onsets ${ }^{254-258}$. In individuals with $\mathrm{PD}$, these sleep-wake symptoms often precede the development of motor or cognitive symptoms and may even be useful as a diagnostic biomarker ${ }^{259,260}$, whereas in patients with $\mathrm{AD}$, sleep disruptions tend to begin after diagnosis ${ }^{261}$. AD brains show marked loss of neurons in the $\mathrm{SCN}^{243,244,262}$, and SCN neuronal loss correlates with reductions in the amplitude of motor activity rhythms in the same individuals before death ${ }^{240}$. In addition, although individuals with AD still show cycling clock gene expression in multiple brain regions, they lose typical phase coherence within and across regions ${ }^{263}$.

In mice, SCN levels of sirtuin 1 (SIRT1), a metabolic sensing protein and mediator of CLOCK and BMAL1 function (FIG. 1), decline with age, potentially explaining the increase in circadian rhythm disruptions in older people $^{241}$. Indeed, brain-specific knockout of Sirt1 in rodents is sufficient to phenocopy the effects of ageing on the SCN and circadian behaviour, whereas overexpression of Sirt1 is protective ${ }^{241}$. Recent studies revealed that SIRT1 binds to CLOCK at the promoter of the gene encoding tyrosine hydroxylase (TH) in dopaminergic neurons to control rhythms in TH expression and, ultimately, dopamine synthesis ${ }^{264}$ (FIG. 5). When iron is present in the cytosol (as may occur more frequently with ageing) ${ }^{265,266}$, dopamine can be oxidized into dopamine quinone (DAQ), which is highly reactive and toxic $^{267,268}$. DAQ has been found in human induced pluripotent stem cell-derived dopaminergic neurons that carry mutations associated with $\mathrm{PD}^{269}$. Thus, deficits in SIRT1 may contribute to toxic levels of DAQ and neurodegeneration.

$\mathrm{PD}$, in particular, is strongly associated with rapid eye moment (REM) sleep behaviour disorder (RBD), as well as excessive daytime sleepiness, insomnia and restless legs syndrome $e^{270-273}$. In fact, more than $80 \%$ of people with $\mathrm{RBD}$ are later diagnosed with $\mathrm{PD}$ or dementia ${ }^{27,275}$. Transgenic mice overexpressing a-synuclein (a model of PD) show greater fragmentation and amplitude reduction in locomotor activity rhythms during the active phase, and these effects progress with age $^{276}$. SCN neuronal firing is also reduced in these mice from young adulthood and progressively worsens with age, suggesting a weakened circadian pacemaker ${ }^{276}$ and a potential loss of dopaminergic modulation of circadian rhythms via midbrain, striatal and SCN circuits $^{277,278}$. MitoPark transgenic mice also show a profound disruption of locomotor rhythms in constant conditions and are more vulnerable to changes in the light-dark cycle ${ }^{279}$.

Sundowning syndrome is prevalent in people with dementia and is characterized by increases in aggression, restlessness, delirium and agitation specifically in the late afternoon or early evening ${ }^{280,281}$. Clinical and preclinical data suggest that a lack of appropriate light-dark cues, disturbances in sleep (particularly, reductions in REM sleep) and a deterioration of the SCN pacemaker and its outputs all contribute to sundowning syndrome ${ }^{281}$. Phase delays and reductions in the amplitude of body temperature rhythms correlate with the severity of sundowning symptoms, suggesting that a breakdown of the circadian system may be responsible ${ }^{282}$. Aggression, for example, may be modulated by the circadian system. A recent study in mice revealed that rhythms in the propensity of aggressive behaviours are modulated via a polysynaptic circuit from VIP neurons in the SCN to GABAergic neurons of the subparaventricular zone, which in turn innervate the ventromedial hypothalamus, an area known to regulate aggression ${ }^{283}$. Further studies are needed to fully elucidate the biological mechanisms that underlie this syndrome, which affects many elderly people and their families.

Several processes regulated by the circadian clock could contribute to neurodegeneration, including the regulation of oxidative stress, inflammation, dopamine synthesis and cellular metabolism. One simple hypothesis is that disruptions in circadian rhythms disrupt sleep, in turn resulting in reduced clearance of misfolded and aggregated proteins that contribute to neurodegenerative disorders from the brain. A recent study found that even one night of sleep deprivation led to accumulation of amyloid- $\beta$ ( $A \beta$; one of the proteins that is pathologically accumulated in the brain in $\mathrm{AD}$ ) in the right hippocampus and thalamus in the human brain ${ }^{284}$. These increases were negatively correlated with mood but were not associated with a particular AD-linked genotype (that is, the $A P O E$ genotype). In line with the clearance hypothesis, during sleep, the glymphatic system ${ }^{285}$ exchanges cerebrospinal fluid with interstitial fluid and clears $A \beta^{286}$. Sleep deprivation increases interstitial fluid levels of $A \beta$ and increases plaque formation in mice and flies ${ }^{287,288}$, whereas in Drosophila, genetically increasing sleep reduces $\mathrm{A} \beta$ deposition ${ }^{288}$. However, based on estimates of anatomical and fluid dynamic parameters, the plausibility of periarterial flow and glymphatic circulation is controversial; thus, further characterization of this system is needed ${ }^{289}$.

The build-up and expulsion of reactive oxygen species (ROS) also follow a circadian pattern. In diurnal species (including humans), ROS accumulate during 


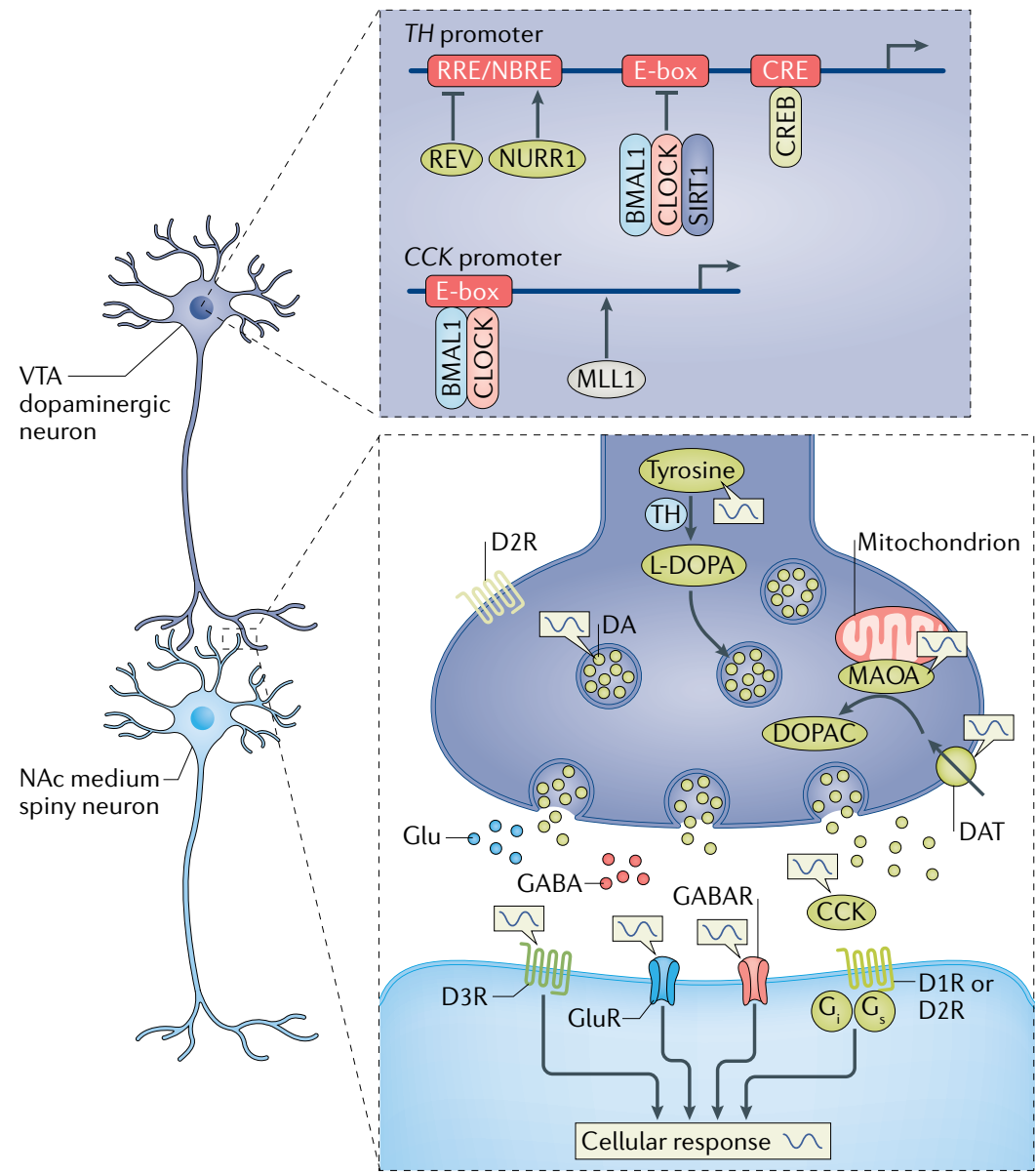

Fig. 5 | Circadian regulation of dopamine. The ventral tegmental area (VTA)-nucleus accumbens (NAc) circuitry is regulated by a local molecular clock, which controls the transcription of genes involved in the dopamine (DA) signalling pathway, including those encoding: tyrosine hydroxylase $(\mathrm{TH})$, the major rate-limiting enzyme converting tyrosine to the DA precursor L-dihydroxyphenylalanine (L-DOPA); cholecystokinin (CCK), a neuropeptide released from presynaptic DA terminals to suppress further DA release; and monoamine oxidase $\mathrm{A}(\mathrm{MAOA})$, a mitochondrial enzyme used to metabolize monoamine neurotransmitters, including DA. For example, the circadian transcription factors of circadian locomotor output cycles protein kaput (CLOCK) and brain and muscle ARNT-like 1 (BMAL1) form heterodimers and recruit the metabolic sensor and histone acetylase sirtuin 1 (SIRT1) to the enhancer box (E-box) within the $T H$ promoter to repress transcription by cAMP response element-binding protein (CREB). CREB activates $\mathrm{TH}$ transcription via the CRE site, located proximal to the transcription start site and the circadian E-box. In addition, CLOCK is capable of binding the E-box within the Cck promoter to promote the transcription of $C c k$ in the VTA. Mixed lineage leukaemia protein 1 (MLL1) is also recruited to CLOCK-BMAL1 heterodimers to promote the transcription of target genes via histone acetylation. REV-ERBa (REV) also represses transcription by binding the REV-ERB response element (RRE) promoter site in antiphase with the transcription factor NURR1 at the NURR1 binding motif, NBRE. Presynaptic dopamine 2 receptors (D2Rs) may also be regulated by the molecular clock. Diurnal rhythms of dopamine, glutamate and GABA levels, as well as of the expression and activity of their receptors, are present in the NAc, potentially temporally gating and promoting neuronal responses during certain times of day. DAT, DA transporter; DOPAC, 3-4-dihydroxyphenylacetic acid; GABAR, GABA receptor; Glu, glutamate; GluR, glutamate receptor (ionotropic).

Astrocytosis

An abnormal increase in the number of astrocytes, attributable to the death of nearby neurons. the day owing to neuronal activity while awake; at night, antioxidants safely remove excess ROS during sleep ${ }^{236,290}$. $\mathrm{Bmal1}^{-{ }^{-}}$mice, which lack a functional molecular clock, show premature ageing, neurodegeneration and a reduced lifespan ${ }^{291}$. ROS accumulation is considerably higher in $\mathrm{Bmal1}^{-/-}$mice and correlates with increases in astrocytosis and levels of oxidative damage markers in the brain. Treating $\mathrm{Bmall}^{-1-}$ mice with the antioxidant $\mathrm{N}$-acetyl-L-cysteine partially rescues their lifespan and prevents age-dependent pathology ${ }^{292}$. Interestingly, mice with a brain-specific Bmall deletion also exhibit increased astrocytosis, despite having mostly normal locomotor circadian rhythms and sleep-wake cycling (that is, relatively normal clock function in the SCN); thus, these neurodegenerative effects are probably due to loss of the local control of cellular metabolism in specific regions of the brain that is normally mediated by BMAL1, independently or as part of the molecular clock, in these cells ${ }^{291}$.

Whether circadian rhythm and sleep disruptions are primarily causal to neurodegenerative disorders remains unclear, but environmental and biological disruptions to these systems do seem to worsen progression of these diseases ${ }^{293-295}$. Thus, therapies that stabilize circadian and sleep rhythms may at least slow the progression of these disorders. Indeed, some studies indicate that morning light therapy may improve circadian rhythms and Mini-Mental State Examination (MMSE), Cohen-Mansfield Agitation Inventory (CMAI) and Behaviour Pathology in AD Rating Scale (BEHAVEAD) scores, particularly in the early stages of dementia, and that, even in severe cases of dementia, it improves circadian and behavioural symptoms, most notably agitation and overall cognitive function ${ }^{296,297}$. Care centres for patients with dementia are often dimly lit ${ }^{298-300}$, and a randomized control trial of 189 participants found that increasing illumination levels in elderly care centres during daytime hours leads to a slower decline in MMSE scores, reduced depression scores and reduced functional impairment in individuals with dementia over a 1.5 -year period ${ }^{301}$. Moreover, a double-blind, multicentre study in 157 individuals with AD showed that evening melatonin treatment improved cognition, decreased nocturnal activity and increased sleep ${ }^{302}$. Importantly, melatonin treatment must be carefully timed to that of normal endogenous melatonin synthesis, as other studies that provided melatonin later in the evening did not find beneficial effects ${ }^{302,303}$.

\section{Conclusions}

Throughout the lifespan, sleep and circadian rhythm disruptions are strongly linked to the pathophysiology of specific psychiatric and neurodegenerative disorders. Though most clinical studies are still correlational, animal model studies are beginning to examine potential causal relationships between disrupted circadian rhythms and multiple brain disorders and are starting to identify molecular mechanisms. Although the central clock in the SCN seems to be key, circadian genes in other brain regions outside of this central pacemaker also contribute locally to the control of neuronal metabolism, neurotransmitter synthesis and activity, and the disruption of these functions might contribute to brain disorders ${ }^{264,304-315}$. We are only beginning to understand the role of peripheral oscillators in multiple regions of the brain, how they are entrained and what other functions circadian transcription factors have that might be independent of the control of 


\section{Mini-Mental State \\ Examination \\ (MMSE). A standardized set of \\ 11 questions with a maximal \\ score of 30 ( $\leq 23$ indicating \\ cognitive impairment) used to \\ assess five cognitive functional areas: orientation, registration, attention and calculation, recall and language.}

daily rhythms. These points should certainly be the focus of future study.

We need to better understand the bidirectional relationships between circadian rhythms and environmental perturbations, such as stress or drugs of abuse, and how circadian rhythms are intimately connected to neurotransmission, metabolism, immunity and other processes. Circadian disruptions may promote vulnerability to or the progression of certain disorders, within a developmental context. With development, changes occur in the sensitivity of the brain to light input, in the inputs and outputs of the SCN, and in the functions of the molecular clock in specific cell types in the brain and in other tissues. In later life, certain disorders exhibit a defined trajectory, with sleep and circadian problems often preceding the development of other symptoms. These findings provide an opening for future, in-depth investigation of the causal relationships between sleep and/or circadian disruption and neurodegeneration.

Recent studies have demonstrated that elements of the molecular circadian clock directly regulate cellular metabolism and mitochondrial function in the liver and other tissues ${ }^{316-318}$. Further studies should determine the molecular mechanisms by which circadian genes may control the metabolism, mitochondrial function, redox state, antioxidant response and activity of neurons and glia, as dysfunction of each of these processes and states can contribute to neurodegeneration. In addition, future studies should determine the circadian rhythm of the expression of drug targets. For example, drugs that target certain neurotransmitters and their receptors may have higher therapeutic efficacy or more adverse effects depending on the time of day the medication is administered and on the endogenous rhythm of neurotransmission and receptor expression.

The evidence for associations between circadian disruptions and brain disorders in humans is mostly epidemiological and correlational. Studies in preclinical models have supported these findings while also offering insights into the bidirectionality, in which circadian disruption leads to certain disease-related phenotypes, and the environmental factors that can alter rhythms, such as stress or exposure to drugs of abuse, can lead to the exacerbation or progression of certain symptoms. Future research will require the continuation of experimental investigation of causality using translational approaches. For example, investigators are beginning to implement laboratory manipulations in humans to misalign the environmental schedule with an individual's endogenous sleep-wake and melatonin rhythms to assess the impact of circadian alignment on cognitive, reward and memory functions, as well as their related neural circuits. Considering these hypotheses within a developmental context will be necessary, as certain developmental periods (for example, adolescence) may be vulnerable to these disruptions. Larger-scale, more in-depth assessments of chronotype utilizing novel biological assays ${ }^{319}$, combined with longitudinal follow-ups, could further clarify the predictive associations between eveningness or delayed chronotypes and brain disorders such as depression, bipolar disorder and addiction. Recent genome-wide association studies with more than 100,000 subjects have identified novel loci associated with sleep duration, chronotype and metabolic phenotypes (for example, body mass index) ) $^{320,321}$. The role of these environmental and genetic factors underlying circadian rhythms and brain disorders can be further experimentally interrogated using cell-based assays and preclinical models. Manipulation of specific circadian genes within neural cell types and regions will begin to dissect the involvement of molecular and cellular clocks in neural circuits and relevant physiological and behavioural end points. Optogenetic and chemogenetic tools can be used to tease apart the function of light-input pathways to the brain that are dependent on, or independent from, SCN connections in regulating neural activity, as has recently been demonstrated for mood-related behaviours ${ }^{322-324}$. With the advent of new tools and resources in neuroscience, and building on a greater understanding of the relationships between circadian rhythms, physiology and behaviour, research in humans and animals is equipped to investigate causal relationships between circadian disruption and various brain disorders. These findings may lead to effective therapeutics and interventions with improved clinical outcomes.

Chronotherapeutic strategies of specifically timing the delivery of certain drugs are now being used in the treatment of certain cancers ${ }^{325}$ and may be therapeutically beneficial across various diseases ${ }^{326}$. Timerestricted feeding is also now being used to help treat and/or prevent obesity, as well as metabolic, cardiac and liver disorders ${ }^{327,328}$. Several first-line pharmacological treatments for psychiatric disorders, such as lithium for bipolar disorder and SSRIs for major depressive disorders, modulate the circadian clock $^{216,217,329-331}$. Although these effects were identified later, they suggest that targeting rhythms or components of the clock using pharmacological agents might be therapeutically beneficial ${ }^{215,216,331}$. Many of the most effective treatments for brain disorders and other diseases modulate clock function ${ }^{216,326,330,332,333}$. Importantly, treatments and interventions that target the circadian system may have therapeutic efficacy and improve clinical symptoms and daily functioning for the individual. Such approaches, if considered, could even be added as adjunct therapies to first-line medications or treatments to further improve therapeutic outcomes. There is much interest in the implementation of regular social, sleep and activity schedules in children and adolescents with developmental disorders, as stable environmental schedules seem to ameliorate daytime sleepiness and daytime behavioural problems in individuals with ASD or ADHD. The increased use of wearable activity and sleep-tracking devices has also brought about new possibilities in terms of diagnostic analysis and personalized treatment plans ${ }^{334}$. The field is poised to align multiple approaches, including tracking of brain function, receptor levels and internal rhythms, that could be valuable for treating psychiatric and neurological disorders in the future.

Published online 20 November 2018 
1. Reppert, S. M. \& Weaver, D. R. Coordination of circadian timing in mammals. Nature 418, 935-941 (2002).

2. Colwell, C. S. Linking neural activity and molecular oscillations in the SCN. Nat. Rev. Neurosci. 12, 553-569 (2011)

3. Selemon, L. D. A role for synaptic plasticity in the adolescent development of executive function. Trans/ Psychiatry 3, e238 (2013)

4. Mighdoll, M. I., Tao, R., Kleinman, J. E. \& Hyde, T. M. Myelin, myelin-related disorders, and psychosis. Schizophr. Res. 161, 85-93 (2015).

5. Hsu, P. K., Ptacek, L. J. \& Fu, Y. H. Genetics of human sleep behavioral phenotypes. Methods Enzymol. 552 309-324 (2015)

6. Roenneberg, T. \& Merrow, M. Entrainment of the human circadian clock. Cold Spring Harb. Symp. Quant. Biol. 72, 293-299 (2007).

7. Foster, R. G. et al. Sleep and circadian rhythm disruption in social jetlag and mental illness. Prog. Mol. Biol. Trans/ Sci. 119, 325-346 (2013).

8. Werner, H., Lebourgeois, M. K., Geiger, A. \& Jenni, O. C. Assessment of chronotype in four- to eleven-year-old children: reliability and validity of the Children's Chronotype Questionnaire (CCTQ). Chronobiol. Int. 26, 992-1014 (2009).

9. Simpkin, C. T. et al. Chronotype is associated with the timing of the circadian clock and sleep in toddlers. J. Sleep Res. 23, 397-405 (2014).

10. Crowley, S. J. et al. A longitudinal assessment of sleep timing, circadian phase, and phase angle of entrainment across human adolescence. PLOS ONE 9, e112199 (2014)

11. Chen, C. Y. et al. Effects of aging on circadian patterns of gene expression in the human prefrontal cortex. Proc. Natl Acad. Sci. USA 113, 206-211 (2015). This is the largest-scale study of circadian rhythms of gene expression in human post-mortem brain tissue, revealing age-related decline in the expression of core circadian genes and the emergence of other rhythmic pathways in older subjects.

12. Cornelissen, G. \& Otsuka, K. Chronobiology of aging: a mini-review. Gerontology 63, 118-128 (2017).

13. Drake, C. L., Roehrs, T., Richardson, G., Walsh, J. K. $\&$ Roth, T. Shift work sleep disorder: prevalence and consequences beyond that of symptomatic day workers. Sleep 27, 1453-1462 (2004).

14. Ramin, C. et al. Night shift work at specific age ranges and chronic disease risk factors. Occup. Environ. Med 72, 100-107 (2015)

15. Bedrosian, T. A. \& Nelson, R. J. Timing of light exposure affects mood and brain circuits. Transl Psychiatry 7, e1017 (2017).

16. Bedrosian, T. A. $\&$ Nelson, R. J. Influence of the modern light environment on mood. Mol. Psychiatry 18, 751-757 (2013).

17. Nakata, A. et al. Association of sickness absence with poor sleep and depressive symptoms in shift workers. Chronobiol. Int. 21, 899-912 (2004).

18. Roth, T. Shift work disorder: overview and diagnosis. J. Clin. Psychiatry 73, e09 (2012).

19. Wright, K. P. Jr, Bogan, R. K. \& Wyatt, J. K. Shift work and the assessment and management of shift work disorder (SWD). Sleep Med. Rev. 17, 41-54 (2012).

20. Benca, R. et al. Biological rhythms, higher brain function, and behavior: gaps, opportunities, and challenges. Brain Res. Rev. 62, 57-70 (2009).

21. Poggiogalle, E., Jamshed, H. \& Peterson, C. M. Circadian regulation of glucose, lipid, and energy metabolism in humans. Metabolism 84, 11-27 (2018)

22. Chaudhari, A., Gupta, R., Makwana, K. \& Kondratov, R. Circadian clocks, diets and aging. Nutr. Healthy Aging 4, 101-112 (2017)

23. Rivkees, S. A. Developing circadian rhythmicity in infants. Pediatr. Endocrinol. Rev. 1, 38-45 (2003).

24. Swaab, D. F. Development of the human hypothalamus. Neurochem. Res. 20, 509-519 (1995).

25. VanDunk, C., Hunter, L. A. \& Gray, P. A. Development, maturation, and necessity of transcription factors in the mouse suprachiasmatic nucleus. J. Neurosci. 31 6457-6467 (2011)

26. Reppert, S. M. Pre-natal development of a hypothalamic biological clock. Progress Brain Res. 93, 119-131; discussion 132 (1992).

27. Reppert, S. M. Interaction between the circadian clocks of mother and fetus. Ciba Found. Symp. 183, 198-207; discussion 207-111 (1995).

28. Reppert, S. M., Weaver, D. R. \& Rivkees, S. A. Maternal communication of circadian phase to the developing mammal. Psychoneuroendocrinology 13 , 63-78 (1988).
29. Shearman, L. P., Zeitzer, J. \& Weaver, D. R. Widespread expression of functional $D_{1}$-dopamine receptors in fetal rat brain. Brain Res. Dev. Brain Res. 102, 105-115 (1997).

30. Reiter, R. J., Tan, D. X., Korkmaz, A. \& Rosales-Corral, S. A. Melatonin and stable circadian rhythms optimize maternal, placental and fetal physiology. Hum. Reprod. Update 20, 293-307 (2014).

31. Okatani, Y. et al. Maternal-fetal transfer of melatonin in pregnant women near term. J. Pineal Res. 25 , 129-134 (1998).

32. Schenker, S. et al. Antioxidant transport by the human placenta. Clin. Nutr. 17, 159-167 (1998).

33. Davis, F. C. \& Mannion, J. Entrainment of hamster pup circadian rhythms by prenatal melatonin injections to the mother. Am. J. Physiol. 255, R439-R448 (1988)

34. Seron-Ferre, M. et al. Circadian rhythms in the fetus. Mol. Cell. Endocrinol. 349, 68-75 (2012).

35. Seron-Ferre, M., Riffo, R., Valenzuela, G. J. \& Germain, A. M. Twenty-four-hour pattern of cortisol in the human fetus at term. Am. J. Obstet. Gynecol. 184, 1278-1283 (2001)

36. Kennaway, D. J., Goble, F. C. \& Stamp, G. E. Factors influencing the development of melatonin rhythmicity in humans. J. Clin. Endocrinol. Metab. 81 1525-1532 (1996).

37. Bisanti, L, Olsen, J., Basso, O. Thonneau, P. $\&$ Karmaus, W. Shift work and subfecundity: a European multicenter study. European Study Group on Infertility and Subfecundity. J. Occup. Environ. Med. 38, 352-358 (1996).

38. Aspholm, R. et al. Spontaneous abortions among Finnish flight attendants. J. Occup. Environ. Med. 41 , 486-491 (1999)

39. Cone, J. E., Vaughan, L. M., Huete, A. \& Samuels, S. J. Reproductive health outcomes among female flight attendants: an exploratory study. J. Occup. Environ. Med. 40, 210-216 (1998).

40. Mahoney, M. M. Shift work, jet lag, and female reproduction. Int. J. Endocrinol. 2010, 813764 (2010).

41. Varcoe, T J J et al. Characterisation of the maternal response to chronic phase shifts during gestation in the rat: implications for fetal metabolic programming. PLOS ONE 8, e53800 (2013).

42. Torres-Farfan, C. et al. Maternal melatonin selectively inhibits cortisol production in the primate fetal adrena gland. J. Physiol. 554, 841-856 (2004)

43. Seron-Ferre, M. et al. Impact of chronodisruption during primate pregnancy on the maternal and newborn temperature rhythms. PLOS ONE 8, e57710 (2013).

44. Matsumoto, T. et al. Circadian myometrial and endocrine rhythms in the pregnant rhesus macaque: effects of constant light and timed melatonin infusion. Am. J. Obstet. Gynecol. 165, 1777-1784 (1991).

45. Novakova, M., Sladek, M. \& Sumova, A. Exposure of pregnant rats to restricted feeding schedule synchronizes the SCN clocks of their fetuses under constant light but not under a light-dark regime. J. Biol. Rhythms 25, 350-360 (2010).

46. Vilches, N. et al. Gestational chronodisruption impairs hippocampal expression of NMDA receptor subunits Grin $1 \mathrm{~b} / \mathrm{Grin} 3 \mathrm{a}$ and spatial memory in the adult offspring. PLOS ONE 9, e91313 (2014).

47. Voiculescu, S. E. et al. Behavioral and molecular effects of prenatal continuous light exposure in the adult rat. Brain Res. 1650, 51-59 (2016)

48. Smarr, B. L., Grant, A. D., Perez, L., Zucker, I \& Kriegsfeld, L. J. Maternal and early-life circadian disruption have long-lasting negative consequences on offspring development and adult behavior in mice. Sci. Rep. 7, 3326 (2017).

49. Cisse, Y. M., Russart, K. L. \& Nelson, R. J. Parental exposure to dim light at night prior to mating alters offspring adaptive immunity. Sci. Rep. 7, 45497 (2017).

50. Cisse, Y. M., Russart, K. L. G. \& Nelson, R. J. Depressive-like behavior is elevated among offspring of parents exposed to dim light at night prior to mating. Psychoneuroendocrinology 83, 182-186 (2017).

51. Lunn, R. M. et al. Health consequences of electric lighting practices in the modern world: a report on the National Toxicology Program's workshop on shift work at night, artificial light at night, and circadian disruption. Sci. Total Environ. 607-608, 1073-1084 (2017).

52. Kennaway, D. J., Stamp, G. E. \& Goble, F. C. Development of melatonin production in infants and the impact of prematurity. J. Clin. Endocrinol. Metab. 75, 367-369 (1992)
53. Davis, K. F., Parker, K. P. \& Montgomery, G. L. Sleep in infants and young children: part two: common sleep problems. J. Pediatr. Health Care 18, 130-137 (2004).

54. Dahl, R. E. Sleep, learning, and the developing brain: early-to-bed as a healthy and wise choice for school aged children. Sleep 28, 1498-1499 (2005).

55. Wolke, D., Meyer, R., Ohrt, B. \& Riegel, K. Incidence and persistence of problems at sleep onset and sleep continuation in the preschool period: results of a prospective study of a representative sample in Bavaria [German]. Prax Kinderpsychol. Kinderpsychiatr. 43 331-339 (1994).

56. Gaylor, E. E., Burnham, M. M., Goodlin-Jones, B. L. $\&$ Anders, T. F. A longitudinal follow-up study of young children's sleep patterns using a developmental classification system. Behav. Sleep Med. 3, 44-61 (2005).

57. Touchette, E. et al. Associations between sleep duration patterns and behavioral/cognitive functioning at school entry. Sleep 30, 1213-1219 (2007).

58. Kobayashi, K. et al. Poor toddler-age sleep schedules predict school-age behavioral disorders in a longitudinal survey. Brain Dev. 37, 572-578 (2015).

59. Gregory, A. M. et al. Prospective longitudinal associations between persistent sleep problems in childhood and anxiety and depression disorders in adulthood. J. Abnorm. Child Psychol. 33, 157-163 (2005).

60. Gregory, A. M., Caspi, A., Moffitt, T. E. \& Poulton, R. Sleep problems in childhood predict neuropsychological functioning in adolescence. Pediatrics 123, 1171-1176 (2009).

61. Hysing, M., Sivertsen, B., Garthus-Niegel, S $\&$ Eberhard-Gran, M. Pediatric sleep problems and social-emotional problems. A population-based study. Infant Behav. Dev, 42, 111-118 (2016).

62. Gregory, A. M., Eley, T. C., O'Connor, T. G. \& Plomin, R. Etiologies of associations between childhood sleep and behavioral problems in a large twin sample. J. Am. Acad. Child Adolesc. Psychiatry 43, 744-751 (2004).

63. Bendova, Z., Sumova, A. \& Illnerova, H. Development of circadian rhythmicity and photoperiodic response in subdivisions of the rat suprachiasmatic nucleus. Brain Res. Dev. Brain Res. 148, 105-112 (2004).

64. Duncan, M. J., Banister, M. J. \& Reppert, S. M Developmental appearance of light-dark entrainment in the rat. Brain Res. 369, 326-330 (1986).

65. Fahrenkrug, J., Nielsen, H. S. \& Hannibal, J. Expression of melanopsin during development of the rat retina. Neuroreport 15, 781-784 (2004).

66. Gonzalez-Menendez, I., Contreras, F., CernudaCernuda, R. \& Garcia-Fernandez, J. M. Daily rhythm of melanopsin-expressing cells in the mouse retina. Front. Cell Neurosci. 3, 3 (2009).

67. Landgraf, D., Koch, C. E. \& Oster, H. Embryonic development of circadian clocks in the mammalian suprachiasmatic nuclei. Front. Neuroanat. 8, 143 (2014).

68. Leard, L. E., Macdonald, E. S., Heller, H. C. \& Kilduff, T. S. Ontogeny of photic-induced c-fos mRNA expression in rat suprachiasmatic nuclei. Neuroreport 5, 2683-2687 (1994)

69. Tarttelin, E. E. et al. Expression of opsin genes early in ocular development of humans and mice. Exp. Eye Res. 76, 393-396 (2003)

70. Rivkees, S. A. The development of circadian rhythms: from animals to Humans. Sleep Med. Clin. 2, 331-341 (2007).

71. Guyer, C. et al. Very preterm infants show earlier emergence of 24-hour sleep-wake rhythms compared to term infants. Early Hum. Dev. 91, 37-42 (2015).

72. Boivin, D. B., Duffy, J. F., Kronauer, R. E. \& Czeisler, C. A Dose-response relationships for resetting of human circadian clock by light. Nature 379, 540-542 (1996).

73. Rivkees, S. A., Hofman, P. L. \& Fortman, J. Newborn primate infants are entrained by low intensity lighting. Proc. Natl Acad. Sci. USA 94, 292-297 (1997).

74. Shanahan, T. L. \& Czeisler, C. A. Physiological effects of light on the human circadian pacemaker. Semin. Perinatol. 24, 299-320 (2000).

75. Watanabe, S. et al. Designing artificial environments for preterm infants based on circadian studies on pregnant uterus. Front. Endocrinol. 4, 113 (2013).

76. Morag, I. \& Ohlsson, A. Cycled light in the intensive care unit for preterm and low birth weight infants. Cochrane Database Syst. Rev. 8, CD006982 (2013).

77. Vasquez-Ruiz, S. et al. A light/dark cycle in the NICU accelerates body weight gain and shortens time to discharge in preterm infants. Early Hum. Dev. 90, 535-540 (2014). 
78. Mirmiran, M. \& Ariagno, R. L. Influence of light in the $\mathrm{NICU}$ on the development of circadian rhythms in preterm infants. Semin. Perinatol. 24, 247-257 (2000).

79. Boo, N. Y., Chee, S. C. \& Rohana, J. Randomized controlled study of the effects of different durations of light exposure on weight gain by preterm infants in a neonatal intensive care unit. Acta Paediatr. 91, 674-679 (2002)

80. Brandon, D. H., Holditch-Davis, D. \& Belyea, M. Preterm infants born at less than 31 weeks' gestation have improved growth in cycled light compared with continuous near darkness. J. Pediatr. 140, 192-199 (2002).

This randomized intervention study reports that preterm infants $(<31$ weeks) receiving cycled light (11 hours on and 11 hours off, with 1 transition hour for shift changes) during hospital care ( $\sim 4$ weeks) gained weight faster than age-matched infants receiving near darkness.

81. Guyer, C. et al. Cycled light exposure reduces fussing and crying in very preterm infants. Pediatrics 130 , e145-e151 (2012)

82. Hao, H. \& Rivkees, S. A. The biological clock of very premature primate infants is responsive to light. Proc. Natl Acad. Sci. USA 96, 2426-2429 (1999).

83. Lebel, V., Aita, M., Johnston, C., Heon, M. \& Dupuis, F. Effects of cycled lighting versus continuous near darkness on physiological stability and motor activity level in preterm infants. Adv. Neonatal Care 17 282-291 (2017).

84. Mann, N. P., Haddow, R., Stokes, L., Goodley, S $\&$ Rutter, N. Effect of night and day on preterm infants in a newborn nursery: randomised trial. Br. Med. J. (Clin. Res. Ed.) 293, (1265-1267 (1986).

85. Rivkees, S. A., Mayes, L., Jacobs, H. \& Gross, I. Rest-activity patterns of premature infants are regulated by cycled lighting. Pediatrics $113,833-839$ (2004).

86. Philipsen, A., Hornyak, M. \& Riemann, D. Sleep and sleep disorders in adults with attention deficit/ hyperactivity disorder. Sleep Med. Rev. 10, 399-405 (2006).

87. Coogan, A. N. \& McGowan, N. M. A systematic review of circadian function, chronotype and chronotherapy in attention deficit hyperactivity disorder. Atten. Defic. Hyperact. Disord. 9, 129-147 (2017).

88. Rybak, Y. E., McNeely, H. E., Mackenzie, B. E., Jain, U. R. $\&$ Levitan, R. D. Seasonality and circadian preference in adult attention-deficit/hyperactivity disorder: clinical and neuropsychological correlates. Compr. Psychiatry 48, 562-571 (2007).

89. Baird, A. L., Coogan, A. N., Siddiqui, A., Donev, R. M. $\&$ Thome, J. Adult attention-deficit hyperactivity disorder is associated with alterations in circadian rhythms at the behavioural, endocrine and molecular levels. Mol. Psychiatry 17, 988-995 (2012).

90. Fargason, R. E. et al. Correcting delayed circadian phase with bright light therapy predicts improvement in ADHD symptoms: a pilot study. J. Psychiatr. Res. 91, 105-110 (2017).

91. Molina-Carballo, A et al. Methylphenidate effects on blood serotonin and melatonin levels may help to synchronise biological rhythms in children with ADHD. J. Psychiatr. Res. 47, 377-383 (2013).

92. Wang, S. M. et al. Modafinil for the treatment of attention-deficit/hyperactivity disorder: a meta-analysis. J. Psychiatr. Res. 84, 292-300 (2017).

93. Gerrard, P. \& Malcolm, R. Mechanisms of modafinil: a review of current research. Neuropsychiatr. Dis. Treat. 3, 349-364 (2007)

94. Cortesi, F., Giannotti, F., Ivanenko, A. \& Johnson, K. Sleep in children with autistic spectrum disorder. Sleep Med. 11, 659-664 (2010).

95. Richdale, A. L. \& Schreck, K. A. Sleep problems in autism spectrum disorders: prevalence, nature, and possible biopsychosocial aetiologies. Sleep Med. Rev. 13, 403-411 (2009)

96. Giannotti, F. et al. An investigation of sleep characteristics, EEG abnormalities and epilepsy in developmentally regressed and non-regressed children with autism. J. Autism Dev. Disord. 38, 1888-1897 (2008).

97. Takase, M., Taira, M. \& Sasaki, H. Sleep-wake rhythm of autistic children. Psychiatry Clin. Neurosci. 52, 181-182 (1998)

98. Hayashi, E. Seasonal changes in sleep and behavioral problems in a pubescent case with autism. Psychiatry Clin. Neurosci. 55, 223-224 (2001).

99. Tordjman, S., Anderson, G. M., Pichard, N., Charbuy, H. \& Touitou, Y. Nocturnal excretion of 6-sulphatoxymelatonin in children and adolescents with autistic disorder. Biol. Psychiatry 57, 134-138 (2005).

100. Nir, I. et al. Brief report: circadian melatonin, thyroidstimulating hormone, prolactin, and cortisol levels in serum of young adults with autism. J. Autism Dev. Disord. 25, 641-654 (1995).

101. Kulman, G. et al. Evidence of pineal endocrine hypofunction in autistic children. Neuro Endocrinol. Lett. 21, 31-34 (2000).

102. Melke, J. et al. Abnormal melatonin synthesis in autism spectrum disorders. Mol. Psychiatry 13, 90-98 (2008).

103. Simonneaux, V. \& Ribelayga, C. Generation of the melatonin endocrine message in mammals: a review of the complex regulation of melatonin synthesis by norepinephrine, peptides, and other pineal transmitters. Pharmacol. Rev. 55, 325-395 (2003).

104. Jin, Y., Choi, J., Won, J. \& Hong, Y. The relationship between autism spectrum disorder and melatonin during fetal development. Molecules 23, E198 (2018).

105. Gringras, P., Nir, T., Breddy, J., Frydman-Marom, A. $\&$ Findling, R. L. Efficacy and safety of pediatric prolonged-release melatonin for insomnia in children with autism spectrum disorder. J. Am. Acad. Child Adolesc. Psychiatry 56, 948-957 (2017).

106. Tordjman, S. et al. Autism as a disorder of biological and behavioral rhythms: toward new therapeutic perspectives. Front. Pediatr. 3, 1 (2015).

107. Hoel, E. P., Albantakis, L., Cirelli, C. \& Tononi, G Synaptic refinement during development and its effect on slow-wave activity: a computational study. J. Neurophysiol. 115, 2199-2213 (2016).

108. Goldstone, A. et al. The mediating role of cortical thickness and gray matter volume on sleep slow-wave activity during adolescence. Brain Struct. Funct. 223. 669-685 (2017).

109. Cassidy, S. B., Schwartz, S., Miller, J. L. \& Driscoll, D. J. Prader-Willi syndrome. Genet. Med. 14, 10-26 (2012).

110. Sahoo, T. et al. Prader-Willi phenotype caused by paternal deficiency for the HBII-85C/D box small nucleolar RNA cluster. Nat. Genet 40, 719-721 (2008).

111. Lassi, G. et al. Deletion of the Snord 116/SNORD 116 alters sleep in mice and patients with Prader-Willi syndrome. Sleep 39, 637-644 (2016).

112. Butler, J. V. et al. Prevalence of, and risk factors for physical ill-health in people with Prader-Willi syndrome a population-based study. Dev. Med. Child Neurol. 44, 248-255 (2002)

113. Cavaille, J. et al. Identification of brain-specific and imprinted small nucleolar RNA genes exhibiting an unusual genomic organization. Proc. Natl Acad. Sci. USA 97, 14311-14316 (2000).

114. Galiveti, C. R., Raabe, C. A., Konthur, Z. \& Rozhdestvensky, T. S. Differential regulation of nonprotein coding RNAs from Prader-Willi syndrome locus. Sci. Rep. 4, 6445 (2014).

115. Runte, M. et al. The IC-SNURF-SNRPN transcript serves as a host for multiple small nucleolar RNA species and as an antisense RNA for UBE3A. Hum Mol. Genet. 10, 2687-2700 (2001).

116. Powell, W. T. et al. A Prader-Willi locus IncRNA cloud modulates diurnal genes and energy expenditure. Hum. Mol. Genet. 22, 4318-4328 (2013).

117. Gossan, N. C. et al. The E3 ubiquitin ligase UBE3A is an integral component of the molecular circadian clock through regulating the BMAL1 transcription factor. Nucleic Acids Res. 42, 5765-5775 (2014).

118. Solter, D. Differential imprinting and expression of maternal and paternal genomes. Annu. Rev. Genet. 22, 127-146 (1988)

119. Martins-Taylor, K. et al. Imprinted expression of UBE3A in non-neuronal cells from a Prader-Willi syndrome patient with an atypical deletion. Hum. Mol Genet. 23, 2364-2373 (2014).

120. Rougeulle, C., Glatt, H. \& Lalande, M. The Angelman syndrome candidate gene, UBE3A/E6-AP, is imprinted in brain. Nat. Genet. 17, 14-15 (1997).

121. Baron, C. A. et al. Genomic and functional profiling of duplicated chromosome 15 cell lines reveal regulatory alterations in UBE3A-associated ubiquitin-proteasome pathway processes. Hum. Mol. Genet. 15, 853-869 (2006).

122. Herzing, L. B., Cook, E. H. Jr \& Ledbetter, D. H Allele-specific expression analysis by RNA-FISH demonstrates preferential maternal expression of UBE3A and imprint maintenance within 15q11-q13 duplications. Hum. Mol. Genet. 11, 1707-1718 (2002).

123. Hogart, A. et al. Chromosome 15q11-13 duplication syndrome brain reveals epigenetic alterations in gene expression not predicted from copy number. J. Med. Genet. 46, 86-93 (2009).

124. Nishimura, Y. et al. Genome-wide expression profiling of lymphoblastoid cell lines distinguishes different forms of autism and reveals shared pathways. Hum. Mol. Genet. 16, 1682-1698 (2007).

125. Dindot, S. V., Antalffy, B. A., Bhattacharjee, M. B. $\&$ Beaudet, A. L. The Angelman syndrome ubiquitin ligase localizes to the synapse and nucleus, and maternal deficiency results in abnormal dendritic spine morphology. Hum. Mol. Genet. 17, 111-118 (2008).

126. Yamasaki, K. et al. Neurons but not glial cells show reciprocal imprinting of sense and antisense transcripts of Ube3a. Hum. Mol. Genet. 12, 837-847 (2003).

127. Shi, S. Q., Bichell, T. J., Ihrie, R. A. \& Johnson, C. H. Ube3a imprinting impairs circadian robustness in Angelman syndrome models. Curr. Biol. 25, 537-545 (2015).

128. Boudreau, E. A. et al. Review of disrupted sleep patterns in Smith-Magenis syndrome and normal melatonin secretion in a patient with an atypical interstitial $17 \mathrm{p} 11.2$ deletion. Am. J. Med. Genet. A 149A, 1382-1391 (2009).

129. Greenberg, F. et al. Multi-disciplinary clinical study of Smith-Magenis syndrome (deletion 17p 11.2). Am. J. Med. Genet. 62, 247-254 (1996).

130. Gropman, A. L., Elsea, S., Duncan, W. C. Jr $\&$ Smith, A. C. New developments in Smith-Magenis syndrome (del 17p 11.2). Curr. Opin. Neurol. 20, 125-134 (2007).

131. Smith, A. C., Dykens, E. \& Greenberg, F. Sleep disturbance in Smith-Magenis syndrome (del 17p11.2) Am. J. Med. Genet. 81, 186-191 (1998)

132. Boone, P. M. et al. Abnormal circadian rhythm of melatonin in Smith-Magenis syndrome patients with RAl1 point mutations. Am. J. Med. Genet. A 155A, 2024-2027 (2011).

133. Walz, K. et al. Behavioral characterization of mouse models for Smith-Magenis syndrome and dup(17) (p11.2p11.2). Hum. Mol. Genet. 13, 367-378 (2004).

134. Lacaria, M., Gu, W. \& Lupski, J. R. Circadian abnormalities in mouse models of Smith-Magenis syndrome: evidence for involvement of RAI1. Am. J. Med. Genet. A 161 A, 1561-1568 (2013).

135. Williams, S. R., Zies, D., Mullegama, S. V., Grotewiel, M. S. \& Elsea, S. H. Smith-Magenis syndrome results in disruption of CLOCK gene transcription and reveals an integral role for RAI 1 in the maintenance of circadian rhythmicity. Am. J. Hum Genet 90, 941-949 (2012).

136. De Leersnyder, $H$. et al. $\beta_{1}$-adrenergic antagonists improve sleep and behavioural disturbances in a circadian disorder, Smith-Magenis syndrome. J. Med. Genet. 38, 586-590 (2001).

137. De Leersnyder, $\boldsymbol{H}$. et al. $\beta_{1}$-adrenergic antagonists and melatonin reset the clock and restore sleep in a circadian disorder, Smith-Magenis syndrome. J. Med. Genet. 40, 74-78 (2003)

138. De Leersnyder, H., Claustrat, B., Munnich, A. $\&$ Verloes, A. Circadian rhythm disorder in a rare disease: Smith-Magenis syndrome. Mol. Cell Endocrinol. 252, 88-91 (2006)

139. Elsea, S. H. \& Girirajan, S. Smith-Magenis syndrome. Eur. J. Hum. Genet. 16, 412-421 (2008).

140. Logan, R. W. et al. Impact of sleep and circadian rhythms on addiction vulnerability in adolescents. Biol. Psychiatry 83, 987-996 (2017)

141. Roenneberg, T. et al. A marker for the end of adolescence. Curr. Biol. 14, R1038-R1039 (2004)

142. Hagenauer, M. H. \& Lee, T. M. The neuroendocrine control of the circadian system: adolescent chronotype. Front. Neuroendocrinol. 33, 211-229 (2012).

143. Hagenauer, M. H. \& Lee, T. M. Adolescent sleep patterns in humans and laboratory animals. Horm. Behav. 64, 270-279 (2013).

144. Cain, N. \& Gradisar, M. Electronic media use and sleep in school-aged children and adolescents: a review. Sleep Med. 11, 735-742 (2010).

145. Crowley, S. J., Cain, S. W., Burns, A. C., Acebo, C. $\&$ Carskadon, M. A. Increased sensitivity of the circadian system to light in early/mid-puberty. J. Clin. Endocrinol. Metab. 100, 4067-4073 (2015). This study shows that endogenous melatonin levels of prepubertal children and early adolescents are more sensitive to the suppressive effects of acute light exposure during the evening than melatonin levels in late adolescents.

146. Paruthi, S. et al. Recommended amount of sleep for pediatric populations: a consensus statement of the 
American Academy of Sleep Medicine. J. Clin. Sleep Med. 12, 785-786 (2016).

147. Basch, C. E., Basch, C. H., Ruggles, K. V. \& Rajan, S Prevalence of sleep duration on an average school night among 4 nationally representative successive samples of American high school students, 2007-2013. Prev Chron. Dis. 11, E216 (2014).

148. Touitou, Y. Adolescent sleep misalignment: a chronic jet lag and a matter of public health. J. Physiol. Paris 107, 323-326 (2013).

149. Hasler, B. P. et al. Weekend-weekday advances in sleep timing are associated with altered reward-related brain function in healthy adolescents. Biol. Psychol. 91, 334-341 (2012).

150. Skeldon, A. C., Phillips, A. J. \& Dijk, D. J. The effects of self-selected light-dark cycles and social constraints on human sleep and circadian timing: a modeling approach. Sci. Rep. 7, 45158 (2017).

151. Crowley, S. J. \& Carskadon, M. A. Modifications to weekend recovery sleep delay circadian phase in older adolescents. Chronobiol. Int. 27, 1469-1492 (2010).

152. Wittmann, M., Dinich, J., Merrow, M. \& Roenneberg, T. Social jetlag: misalignment of biological and social time. Chronobiol. Int. 23, 497-509 (2006).

153. Chen, K. \& Kandel, D. B. The natural history of drug use from adolescence to the mid-thirties in a general population sample. Am. J. Publ. Health 85, 41-47 (1995).

154. Falcon, E. \& McClung, C. A. A role for the circadian genes in drug addiction. Neuropharmacology 56 91-96 (2009). (Suppl. 1).

155. O'Brien, E. M. \& Mindell, J. A. Sleep and risk-taking behavior in adolescents. Behav. Sleep Med. 3, 113-133 (2005)

156. Pasch, K. E., Laska, M. N., Lytle, L. A. \& Moe, S. G. Adolescent sleep, risk behaviors, and depressive symptoms: are they linked? Am. J. Health Behav. 34, 237-248 (2010).

157. McKnight-Eily, L. R. et al. Relationships between hours of sleep and health-risk behaviors in US adolescent students. Prev. Med. 53, 271-273 (2011).

158. Paiva, T., Gaspar, T. \& Matos, M. G. Mutual relations between sleep deprivation, sleep stealers and risk behaviours in adolescents. Sleep Sci. 9, 7-13 (2016).

159. Sivertsen, B., Skogen, J. C., Jakobsen, R. \& Hysing, M. Sleep and use of alcohol and drug in adolescence. A large population-based study of Norwegian adolescents aged 16 to 19 years. Drug Alcohol Depend. 149, 180-186 (2015).

160. Thomas, A. G., Monahan, K. C., Lukowski, A. F. ¿ Cauffman, E. Sleep problems across development: a pathway to adolescent risk taking through working memory. J. Youth Adolesc. 44, 447-464 (2015).

161. Wheaton, A. G., Olsen, E. O., Miller, G. F. \& Croft, J. B. Sleep duration and injury-related risk behaviors among high school students - United States, 2007-2013. MMWR Morb. Mortal. Wkly Rep. 65, 337-341 (2016).

162. Pasch, K. E., Latimer, L. A., Cance, J. D., Moe, S. G. \& Lytle, L. A. Longitudinal bi-directional relationships between sleep and youth substance use. J. Youth Adolesc. 41, 1184-1196 (2012)

163. Hasler, B. P., Martin, C. S., Wood, D. S., Rosario, B. \& Clark, D. B. A longitudinal study of insomnia and other sleep complaints in adolescents with and without alcohol use disorders. Alcohol Clin. Exp. Res. 38, 2225-2233 (2014).

164. Hasler, B. P., Kirisci, L. \& Clark, D. B. Restless sleep and variable sleep timing during late childhood accelerate the onset of alcohol and other drug involvement. J. Stud. Alcohol Drugs 77, 649-655 (2016).

165. Hasler, B. P., Soehner, A. M. \& Clark, D. B. Sleep and circadian contributions to adolescent alcohol use disorder. Alcohol 49, 377-387 (2015).

166. Tavernier, R., Munroe, M. \& Willoughby, T. Perceived morningness-eveningness predicts academic adjustment and substance use across university, but social jetlag is not to blame. Chronobiol. Int. 32 , 1233-1245 (2015).

167. Hasler, B. P., Casement, M. D., Sitnick, S. L., Shaw, D. S $\&$ Forbes, E. E. Eveningness among late adolescent males predicts neural reactivity to reward and alcohol dependence two years later. Behav. Brain Res. 327, 112-120 (2017)

This study shows that an evening chronotype during late adolescence ( 20 years of age) is associated with strength of activation in the VS to reward outcome, which was positively correlated with alcohol dependence 2 years later.

168. Mednick, S. C., Christakis, N. A. \& Fowler, J. H. The spread of sleep loss influences drug use in adolescent social networks. PLOS ONE 5, e9775 (2010).
169. Forbes, E. E. et al. Healthy adolescents' neural response to reward: associations with puberty, positive affect, and depressive symptoms. J. Am. Acad. Child Adolesc. Psychiatry 49, 162-172 (2010).

170. Padmanabhan, A., Geier, C. F., Ordaz, S. J., Teslovich, T. \& Luna, B. Developmental changes in brain function underlying the influence of reward processing on inhibitory control. Dev. Cogn. Neurosci. 1, 517-529 (2011).

171. Frischknecht, R. \& Gundelfinger, E. D. The brain's extracellular matrix and its role in synaptic plasticity. Adv. Exp. Med. Biol. 970, 153-171 (2012).

172. Luciana, M. \& Collins, P. F. Incentive motivation, cognitive control, and the adolescent brain: is it time for a paradigm shift? Child Dev. Perspect. 6, 392-399 (2012).

173. Padmanabhan, A. \& Luna, B. Developmental imaging genetics: linking dopamine function to adolescent behavior. Brain Cogn. 89, 27-38 (2014).

174. Ernst, M. \& Fudge, J. L. A developmental neurobiological model of motivated behavior: anatomy, connectivity and ontogeny of the triadic nodes. Neurosci. Biobehav. Rev. 33, 367-382 (2009).

175. Somerville, L. H., Jones, R. M. \& Casey, B. J. A time of change: behavioral and neural correlates of adolescent sensitivity to appetitive and aversive environmental cues. Brain Cogn . 72, 124-133 (2010).

176. Logan, R. W., Williams, W. P. \& McClung, C. A Circadian rhythms and addiction: mechanistic insights and future directions. Behav. Neurosci. 128 387-412 (2014)

177. Hasler, B. P., Forbes, E. E. \& Franzen, P. L. Time-of-day differences and short-term stability of the neural response to monetary reward: a pilot study. Psychiatry Res. 224, 22-27 (2014).

178. Hasler, B. P. et al. Chronotype and diurnal patterns of positive affect and affective neural circuitry in primary insomnia. J. Sleep Res. 21, 515-526 (2012).

179. Hasler, B. P., Sitnick, S. L., Shaw, D. S. \& Forbes, E. E. An altered neural response to reward may contribute to alcohol problems among late adolescents with an evening chronotype. Psychiatry Res. 214, 357-364 (2013).

180. Mullin, B. C. et al. Sleep deprivation amplifies striatal activation to monetary reward. Psychol. Med. 43, 2215-2225 (2013)

181. Miller, M. B., Donahue, M. L., Carey, K. B. $\&$ Scott-Sheldon, L. A. J. Insomnia treatment in the context of alcohol use disorder: a systematic review and meta-analysis. Drug Alcohol Depend. 181, 200-207 (2017).

182. Merikangas, K. R. et al. Lifetime prevalence of mental disorders in U. S. adolescents: results from the National Comorbidity Survey Replication - Adolescent Supplement (NCS-A). J. Am. Acad. Child Adolesc. Psychiatry 49, 980-989 (2010).

183. Lamont, E. W., Coutu, D. L., Cermakian, N. $\&$ Boivin, D. B. Circadian rhythms and clock genes in psychotic disorders. Isr. J. Psychiatry Relat. Sci. 47 , 27-35 (2010)

184. McClung, C. A. How might circadian rhythms control mood? Let me count the ways. Biol. Psychiatry 74 242-249 (2013)

185. Mansour, H. A. et al. Circadian phase variation in bipolar I disorder. Chronobiol. Int. 22, 571-584 (2005).

186. McCarthy, M. J. \& Welsh, D. K. Cellular circadian clocks in mood disorders. J. Biol. Rhythms 27 339-352 (2012).

187. McClung, C. A. Circadian rhythms and mood regulation: insights from pre-clinical models. Eur. Neuropsychopharmacol. 21, S683-S693 (2011).

188. Wulff, K., Dijk, D. J., Middleton, B., Foster, R. G. $\&$ Joyce, E. M. Sleep and circadian rhythm disruption in schizophrenia. Br. J. Psychiatry 200, 308-316 (2012).

This study reports notable circadian misalignment (phase delays and advances) of sleep-wake cycles and melatonin rhythms in patients with schizophrenia despite other factors, including mood, cognitive status and pharmacological treatments.

189. Frank, E., Swartz, H. A. \& Kupfer, D. J. Interpersona and social rhythm therapy: managing the chaos of bipolar disorder. Biol. Psychiatry 48, 593-604 (2000).

190. Malkoff-Schwartz, S. et al. Stressful life events and social rhythm disruption in the onset of manic and depressive bipolar episodes: a preliminary investigation. Arch. Gen. Psychiatry 55, 702-707 (1998)

191. Melo, M. C. A., Abreu, R. L. C., Linhares Neto, V. B. de Bruin, P. F. C. \& de Bruin, V. M. S. Chronotype and circadian rhythm in bipolar disorder: a systematic review. Sleep Med. Rev. 34, 46-58 (2017).

192. Winthorst, W. H. et al. Seasonal affective disorder and non-seasonal affective disorders: results from the NESDA study. BJPsych Open 3, 196-203 (2017).

193. Medici, C. R., Vestergaard, C. H., Hadzi-Pavlovic, D. Munk-Jorgensen, P. $\&$ Parker, G. Seasonal variations in hospital admissions for mania: examining for associations with weather variables over time. J. Affect. Disord. 205, 81-86 (2016)

194. Young, J. W. \& Dulcis, D. Investigating the mechanism(s) underlying switching between states in bipolar disorder. Eur. J. Pharmacol. 759, 151-162 (2015).

195. Raniti, M. B. et al. Sleep duration and sleep quality: associations with depressive symptoms across adolescence. Behav. Sleep Med. 15, 198-215 (2017).

196. Lewy, A. J. Depressive disorders may more commonly be related to circadian phase delays rather than advances: time will tell. Sleep Med. 11, 117-118 (2010).

197. Addington, J. \& Heinssen, R. Prediction and prevention of psychosis in youth at clinical high risk. Annu. Rev. Clin. Psychol. 8, 269-289 (2012).

198. Lunsford-Avery, J. R. et al. Adolescents at clinicalhigh risk for psychosis: circadian rhythm disturbances predict worsened prognosis at 1-year follow-up. Schizophr. Res. 189, 37-42 (2017).

199. Robillard, R. et al. Sleep-wake profiles predict longitudinal changes in manic symptoms and memory in young people with mood disorders. J. Sleep Res. 25, 549-555 (2016).

200. Crews, F. T., Vetreno, R. P., Broadwater, M. A. \& Robinson, D. L. Adolescent alcohol exposure persistently impacts adult neurobiology and behavior Pharmacol. Rev. 68, 1074-1109 (2016).

201. Wei, Y., Krishnan, G. P. \& Bazhenov, M. Synaptic mechanisms of memory consolidation during sleep slow oscillations. J. Neurosci. 36, 4231-4247 (2016).

202. Walker, M. P. \& Stickgold, R. Sleep-dependent learning and memory consolidation. Neuron 44 , 121-133 (2004)

203. Havekes, R., Meerlo, P. \& Abel, T. Animal studies on the role of sleep in memory: from behavioral performance to molecular mechanisms. Curr. Top. Behav. Neurosci. 25, 183-206 (2015).

204. Marshall, L., Helgadottir, H., Molle, M. \& Born, J. Boosting slow oscillations during sleep potentiates memory. Nature 444, 610-613 (2006). This study establishes the importance of slow-wave sleep during non-REM episodes that occur during the early night and shows that it promotes the retention of hippocampus-dependent declarative memories.

205. Selemon, L. D. \& Zecevic, N. Schizophrenia: a tale of two critical periods for prefrontal cortical development. Transl Psychiatry 5, e623 (2015).

206. Keshavan, M. S., Anderson, S. \& Pettegrew, J. W. Is schizophrenia due to excessive synaptic pruning in the prefrontal cortex? The Feinberg hypothesis revisited. J. Psychiatr. Res. 28, 239-265 (1994).

207. Billeh, Y. N. et al. Effects of chronic sleep restriction during early adolescence on the adult pattern of connectivity of mouse secondary motor cortex. eNeuro https://doi.org/10.1523/ENEURO.005316-2016 (2016)

208. Carskadon, M. A. Patterns of sleep and sleepiness in adolescents. Pediatrician 17, 5-12 (1990).

209. Johnson, E. O., Roth, T., Schultz, L. \& Breslau, N. Epidemiology of DSM-IV insomnia in adolescence: lifetime prevalence, chronicity, and an emergent gender difference. Pediatrics 117, e247-e256 (2006).

210. Sivertsen, B. et al. Delayed sleep phase syndrome in adolescents: prevalence and correlates in a large population based study. BMC Publ. Health 13, 1163 (2013).

211. Owens, J. A., Belon, K. \& Moss, P. Impact of delaying school start time on adolescent sleep, mood, and behavior. Arch. Pediatr. Adolesc. Med. 164, 608-614 (2010).

212. Wirz-Justice, A. \& Terman, M. Chronotherapeutics (light and wake therapy) as a class of interventions for affective disorders. Handb. Clin. Neurol. 106, 697-713 (2012)

213. Srinivasan, V., De Berardis, D., Shillcutt, S. D. $\&$ Brzezinski, A. Role of melatonin in mood disorders and the antidepressant effects of agomelatine. Expert Opin. Investig. Drugs 21, 1503-1522 (2012).

214. Wu, J. C. et al. Rapid and sustained antidepressant response with sleep deprivation and chronotherapy in bipolar disorder. Biol. Psychiatry 66, 298-301 (2009). This study demonstrates, in a cohort of 49 patients with bipolar disorder, the ability of non-invasive 
circadian therapy (that is, bright light therapy and sleep phase advance) and sleep (that is, acute sleep deprivation) to improve and sustain the therapeutic efficacy of lithium and antidepressants on mood symptoms.

215. Li, J., Lu, W. Q., Beesley, S., Loudon, A. S. \& Meng, O. J. Lithium impacts on the amplitude and period of the molecular circadian clockwork. PLOS ONE 7, e33292 (2012)

216. Johansson, A. S., Brask, J., Owe-Larsson, B., Hetta, J. \& Lundkvist, G. B. Valproic acid phase shifts the rhythmic expression of Period2::Luciferase. J. BiOl. Rhythms 26, 541-551 (2011)

217. Sprouse, J., Braselton, J. \& Reynolds, L. Fluoxetine modulates the circadian biological clock via phase advances of suprachiasmatic nucleus neuronal firing. Biol Psychiatry 60, 896-899 (2006).

218. Duncan, W. C. Jr et al. Motor-activity markers of circadian timekeeping are related to ketamine's rapid antidepressant properties. Biol. Psychiatry 82 361-369 (2017)

In a study of $\mathbf{5 1}$ patients with major depressive disorder or bipolar disorder, circadian activity patterns prior to and following the administration of ketamine are linked to therapeutic response of mood symptoms.

219. Kragh, M. et al. Predictors of response to combined wake and light therapy in treatment-resistant inpatients with depression. Chronobiol. Int. https://doi.org/ 10.1080/07420528.2018.1468341 (2018)

220. Adan, A. \& Natale, V. Gender differences in morningness-eveningness preference. Chronobiol. Int 19, 709-720 (2002)

221. Caci, H., Deschaux, O., Adan, A. \& Natale, V. Comparing three morningness scales: age and gender effects, structure and cut-off criteria. Sleep Med. 10 240-245 (2009)

222. Duarte, L. L. et al. Chronotype ontogeny related to gender. Braz. J. Med. Biol. Res. 47, 316-320 (2014).

223. Paine, S. J., Gander, P. H. \& Travier, N. The epidemiology of morningness/eveningness: influence of age, gender, ethnicity, and socioeconomic factors in adults (30-49 years). J. Biol. Rhythms 21 , 68-76 (2006)

224. Patke, A. et al. Mutation of the human circadian clock gene $C R Y 1$ in familial delayed sleep phase disorder. Cell 169, 203-215 (2017)

225. Kripke, D. F. et al. Circadian polymorphisms in night owls, in bipolars, and in non-24-hour sleep cycles. Psychiatry Invest. 11, 345-362 (2014).

226. Murray, J. M. et al. Prevalence of circadian misalignment and its association with depressive symptoms in delayed sleep phase disorder. Sleep 40 , zsw002 (2017)

227. James, S. M., Honn, K. A., Gaddameedhi, S \& Van Dongen, H. P. A. Shift work: disrupted circadian rhythms and sleep-implications for health and well-being. Curr. Sleep Med. Rep. 3, 104-112 (2017).

228. Hall, A. L., Franche, R. L. \& Koehoorn, M. Examining exposure assessment in shift work research: a study on depression among nurses. Ann. Work Expo. Health 62, 182-194 (2018)

229. Kang, M. Y., Kwon, H. J., Choi, K. H., Kang, C. W. $\Sigma \mathrm{Kim}, \mathrm{H}$. The relationship between shift work and mental health among electronics workers in South Korea: a cross-sectional study. PLOS ONE 12. e0188019 (2017)

230. McNeely, E. et al. The self-reported health of U. S flight attendants compared to the general population. Environ. Health 13, 13 (2014)

231. McNeely, E. et al. Cancer prevalence among flight attendants compared to the general population. Environ. Health 17, 49 (2018)

232. Grajewski, B., Whelan, E. A., Nguyen, M. M., Kwan, L. $\&$ Cole, R. J. Sleep disturbance in female flight attendants and teachers. Aerosp. Med. Hum. Perform. 87, 638-645 (2016).

233. McNeely, E., Mordukhovich, I., Tideman, S., Gale, S. \& Coull, B. Estimating the health consequences of flight attendant work: comparing flight attendant health to the general population in a cross-sectional study. BMC Publ. Health 18, 346 (2018).

234. Feinsilver, S. H. \& Hernandez, A. B. Sleep in the elderly: unanswered questions. Clin. Geriatr. Med. 33 579-596 (2017)

235. Mattis, J. \& Sehgal, A. Circadian rhythms, sleep, and disorders of aging. Trends Endocrinol. Metab. 27 192-203 (2016).

236. Musiek, E. S. \& Holtzman, D. M. Mechanisms linking circadian clocks, sleep, and neurodegeneration. Science 354, 1004-1008 (2016).
237. Karasek, M. Melatonin, human aging, and age-related diseases. Exp. Gerontol. 39, 1723-1729 (2004).

238. Zeitzer, J. M. et al. Do plasma melatonin concentrations decline with age? Am. J. Med. 107 432-436 (1999)

239. Czeisler, C. A. et al. Association of sleep-wake habits in older people with changes in output of circadian pacemaker. Lancet 340, 933-936 (1992). This landmark study suggests that changes in sleep timing and consolidation driven by the circadian system underlie sleep disturbances in elderly individuals.

240. Wang, J. L. et al. Suprachiasmatic neuron numbers and rest-activity circadian rhythms in older humans. Ann. Neurol. 78, 317-322 (2015).

241. Chang, H. C. \& Guarente, L. SIRT1 mediates centra circadian control in the SCN by a mechanism that decays with aging. Cell 153, 1448-1460 (2013). This mouse study reveals that reductions of sirtuin activity in the SCN leads to a gradual age-dependent dampening of SCN activity and locomotor rhythms.

242. Yamazaki, S. et al. Effects of aging on central and peripheral mammalian clocks. Proc. Natl Acad. Sci. USA 99, 10801-10806 (2002).

243. Swaab, D. F., Fliers, E. \& Partiman, T. S. The suprachiasmatic nucleus of the human brain in relation to sex, age and senile dementia. Brain Res. 342, 37-44 (1985)

244. Zhou, J. N., Hofman, M. A. \& Swaab, D. F. VIP neurons in the human $\mathrm{SCN}$ in relation to sex, age, and Alzheimer's disease. Neurobiol. Aging 16, 571-576 (1995).

245. Roozendaal, B., van Gool, W. A., Swaab, D. F. Hoogendijk, J. E. \& Mirmiran, M. Changes in vasopressin cells of the rat suprachiasmatic nucleus with aging. Brain Res. 409, 259-264 (1987).

246. Tsukahara, S., Tanaka, S., Ishida, K., Hoshi, N. $\&$ Kitagawa, H. Age-related change and its sex differences in histoarchitecture of the hypothalamic suprachiasmatic nucleus of F344/N rats. Exp. Gerontol. 40, 147-155 (2005)

247. Nygard, M. Hill, R. H. Wikstrom, M. A. $\&$ Kristensson, K. Age-related changes in electro physiological properties of the mouse suprachiasmatic nucleus in vitro. Brain Res. Bull. 65 149-154 (2005)

248. Farajnia, S. et al. Evidence for neuronal desynchrony in the aged suprachiasmatic nucleus clock. J. Neurosc 32, 5891-5899 (2012)

249. Nakamura, T. J. et al. Age-related decline in circadian output. J. Neurosci. 31, 10201-10205 (2011).

250. Tranah, G. J. et al. Circadian activity rhythms and risk of incident dementia and mild cognitive impairment in older women. Ann. Neurol. 70, 722-732 (2011).

251. Chen, H. F., Huang, C. O., You, C., Wang, Z. R. ¿ Si-qing, H. Polymorphism of CLOCK gene rs $4580704 \mathrm{C}>\mathrm{G}$ is associated with susceptibility of Alzheimer's disease in a Chinese population. Arch. Med. Res. 44, 203-207 (2013)

252. Chen, Q., Peng, X. D., Huang, C. Q., Hu, X. Y \& Zhang, X. M. Association between ARNTL (BMAL1) rs2278749 polymorphism T>C and susceptibility to Alzheimer disease in a Chinese population. Genet Mol. Res. 14, 18515-18522 (2015)

253. Gu, Z. et al. Association of ARNTL and PER 1 genes with Parkinson's disease: a case-control study of Han Chinese. Sci. Rep. 5, 15891 (2015)

254. Videnovic, A. \& Golombek, D. Circadian dysregulation in Parkinson's disease. Neurobiol. Sleep Circadian Rhythms 2, 53-58 (2017).

255. Breen, D. P et al Sleep and circadian rhythm regulation in early Parkinson disease. JAMA Neurol. 71, 589-595 (2014) This study shows significantly increased sleep latency, reduced sleep efficiency and reduced REM along with reduced melatonin levels and a loss of BMAL1 serum expression rhythms, in a cohort of 239 individuals with PD.

256. Gros, P. \& Videnovic, A. Sleep and circadian rhythm disorders in Parkinson's disease. Curr. Sleep Med. Rep. 3, 222-234 (2017).

257. Uchida, K Okamoto, N. Ohara K. \& Morita Y. Daily rhythm of serum melatonin in patients with dementia of the degenerate type. Brain Res. 717 154-159 (1996).

258. Videnovic, A. et al. Circadian melatonin rhythm and excessive daytime sleepiness in Parkinson disease. JAMA Neurol. 71, 463-469 (2014).

259. Claassen, D. O. \& Kutscher, S. J. Sleep disturbances in Parkinson's disease patients and management options. Nat. Sci. Sleep 3, 125-133 (2011).
260. Rothman S. M \& Mattson, M. P. Sleep disturbances in Alzheimer's and Parkinson's diseases. Neuromolecular Med. 14, 194-204 (2012).

261. Vitiello, M. V., Prinz, P. N., Williams, D. E. Frommlet, M. S. \& Ries, R. K. Sleep disturbances in patients with mild-stage Alzheimer's disease. J. Gerontol. 45, M131-M138 (1990)

262. Stopa, E. G. et al. Pathologic evaluation of the human suprachiasmatic nucleus in severe dementia. J. Neuropathol. Exp. Neurol. 58, 29-39 (1999).

263. Cermakian, N., Lamont, E. W., Boudreau, P. \& Boivin, D. B. Circadian clock gene expression in brain regions of Alzheimer 's disease patients and control subjects. J. Biol. Rhythms 26, 160-170 (2011).

264. Logan, R. W. et al. NAD+ cellular redox and SIRT1 regulate the diurnal rhythms of tyrosine hydroxylase and conditioned cocaine reward. Mol. Psychiatry. https://doi.org/10.1038/s41380-018-0061-1 (2018). This study demonstrates mechanistic links between circadian regulation of SIRT 1-dependent metabolic signalling and dopaminergic neurotransmission in the mouse ventral tegmental area, which is important for drug reward.

265. Belaidi, A. A. et al. Marked age-related changes in brain iron homeostasis in amyloid protein precursor knockout mice. Neurotherapeutics https://doi.org/ 10.1007/s13311-018-0656-x (2018).

266. Uranga, R. M. \& Salvador, G. A. Unraveling the burden of iron in neurodegeneration: intersections with amyloid beta peptide pathology. Oxid. Med. Cell. Longev. 2018, 2850341 (2018).

267. Graham, D. G. Oxidative pathways for catecholamines in the genesis of neuromelanin and cytotoxic quinones. Mol. Pharmacol. 14, 633-643 (1978)

268. Tse, D. C., McCreery, R. L. \& Adams, R. N. Potential oxidative pathways of brain catecholamines. J. Med. Chem. 19, 37-40 (1976).

269. Burbulla, L. F. et al. Dopamine oxidation mediates mitochondrial and lysosomal dysfunction in Parkinson's disease. Science 357, 1255-1261 (2017).

This study demonstrates that mitochondrial stress promotes the accumulation of oxidized dopamine, consequently increasing $\alpha$-synuclein aggregates in neurons derived from patients with PD, an effect selective to human neurons.

270. Barone, P. et al. The PRIAMO study: a multicenter assessment of nonmotor symptoms and their impact on quality of life in Parkinson's disease. Mov. Disord. 24, 1641-1649 (2009)

271. Chaudhuri, K. R. et al. International multicenter pilot study of the first comprehensive self-completed nonmotor symptoms questionnaire for Parkinson's disease: the NMSQuest study. Mov. Disord. 21, 916-923 (2006)

272. Goodman, A. O., Morton, A. J. \& Barker, R. A Identifying sleep disturbances in Huntington's disease using a simple disease-focused questionnaire. PLOS Curr. 2, RRN1189 (2010).

273. Aziz, N. A., Anguelova, G. V., Marinus, J., Lammers, G. J. $\&$ Roos, R. A. Sleep and circadian rhythm alterations correlate with depression and cognitive impairment in Huntington's disease. Parkinsonism Relat. Disord. 16 345-350 (2010)

274. Iranzo, A. et al. Neurodegenerative disorder risk in idiopathic REM sleep behavior disorder: study in 174 patients. PLOS ONE 9, e89741 (2014).

275. Schenck, C. H., Boeve, B. F. \& Mahowald, M. W. Delayed emergence of a parkinsonian disorder or dementia in $81 \%$ of older men initially diagnosed with idiopathic rapid eye movement sleep behavior disorder: a 16-year update on a previously reported series. Sleep Med. 14, 744-748 (2013).

276. Kudo, T., Loh, D. H., Truong, D., Wu, Y. \& Colwell, C. S Circadian dysfunction in a mouse model of Parkinson's disease. Exp. Neurol. 232, 66-75 (2011).

277. Grippo, R. M., Purohit, A. M., Zhang, Q., Zweifel, L. S. $\&$ Guler, A. D. Direct midbrain dopamine input to the suprachiasmatic nucleus accelerates circadian entrainment. Curr. Biol. 27, 2465-2475 (2017) This study shows re-entrainment of the SCN to phase shifts in the light-dark schedule is facilitated by dopaminergic projections from the ventral tegmental area to the $\mathrm{SCN}$ and is dependent on dopamine 1 receptor-expressing neurons in the SCN.

278. Korshunov, K. S., Blakemore, L. J. \& Trombley, P. O. Dopamine: a modulator of circadian rhythms in the central nervous system. Front Cell. Neurosci. 11, 91 (2017).

279. Fifel, K. \& Cooper, H. M. Loss of dopamine disrupts circadian rhythms in a mouse model of Parkinson's disease. Neurobiol. Dis. 71, 359-369 (2014). 
280. Gnanasekaran, G. "Sundowning" as a biological phenomenon: current understandings and future directions: an update. Aging Clin. Exp. Res. 28, 383-392 (2016)

281. Bedrosian, T. A. $\bar{\Sigma}$ Nelson, R. J. Sundowning syndrome in aging and dementia: research in mouse models. Exp. Neurol. 243, 67-73 (2013)

282. Volicer, L., Harper, D. G., Manning, B. C., Goldstein, R. $\&$ Satlin, A. Sundowning and circadian rhythms in Alzheimer's disease. Am. J. Psychiatry 158, 704-711 (2001).

This study shows that patients with AD have less diurnal activity, more nocturnal activity and phase delays in body-temperature rhythms, and that the severity of sundowning is positively correlated with lower-amplitude and more-phase-delayed rhythms.

283. Todd, W. D. et al. A hypothalamic circuit for the circadian control of aggression. Nat. Neurosci. 21, 717-724 (2018)

This chemogenetic study in mice reveals a neural circuit involving projections from the SCN to the ventromedial hypothalamus that drives time-of-day-dependent aggressive behaviours.

284. Shokri-Kojori, E. et al. $\beta$-Amyloid accumulation in the human brain after one night of sleep deprivation. Proc. Natl Acad. Sci. USA 115, 4483-4488 (2018)

285. Xie, L. et al. Sleep drives metabolite clearance from the adult brain. Science 342, 373-377 (2013). A landmark study in mice demonstrating that wakefulness suppresses the outflow of cerebral spinal fluid from the brain, whereas sleep leads to an increase in convective fluxes of fluid, effectively promoting the clearance of neurotoxic metabolics, including $\mathbf{A} \boldsymbol{\beta}$.

286. Da Mesquita, S. et al. Functional aspects of meningeal lymphatics in ageing and Alzheimer's disease. Nature 560, 185-191 (2018).

287. Kang, J. E. et al. Amyloid- $\beta$ dynamics are regulated by orexin and the sleep-wake cycle. Science $\mathbf{3 2 6}$, 1005-1007 (2009)

288. Tabuchi, $M$. et al. Sleep interacts with $A \beta$ to modulate intrinsic neuronal excitability. Curr. Biol. 25, 702-712 (2015).

289. Faghih, M. M. \& Sharp, M. K. Is bulk flow plausible in perivascular, paravascular and paravenous channels? Fluids Barriers CNS 15, 17 (2018).

290. McClung, C. A. Mind your rhythms: an important role for circadian genes in neuroprotection. J. Clin. Invest. 123, 4994-4996 (2013)

291. Musiek, E. S. et al. Circadian clock proteins regulate neuronal redox homeostasis and neurodegeneration. J. Clin. Invest. 123, 5389-5400 (2013). This preclinical investigation demonstrates that components of the molecular clock control redox state and, when disrupted, lead to oxidative stress and cell death related to neurodegeneration

292. Kondratov, R. V., Vykhovanets, O., Kondratova, A. A. $\S$ Antoch, M. P. Antioxidant $N$-acetyl-L-cysteine ameliorates symptoms of premature aging associated with the deficiency of the circadian protein BMAL1. Aging 1, 979-987 (2009).

293. Chauhan, R., Chen, K. F., Kent, B. A. \& Crowther, D. C. Central and peripheral circadian clocks and their role in Alzheimer's disease. Dis. Model. Mech. 10 1187-1199 (2017).

294. Homolak, J., Mudrovcic, M., Vukic, B. \& Toljan, K. Circadian rhythm and Alzheimer's disease. Med. Sci. 6, $52(2018)$

295. Huang, Z. et al. Circadian rhythm dysfunction accelerates disease progression in a mouse model with amyotrophic lateral sclerosis. Front. Neurol. 9 218 (2018).

296. Skjerve, A., Bjorvatn, B. \& Holsten, F. Light therapy for behavioural and psychological symptoms of dementia. Int. J. Geriatr. Psychiatry 19, 516-522 (2004).

297. Yamadera, H. et al. Effects of bright light on cognitive and sleep-wake (circadian) rhythm disturbances in Alzheimer-type dementia. Psychiatry Clin. Neurosci. 54, 352-353 (2000)

298. Hanford, N. \& Figueiro, M. Light therapy and Alzheimer's disease and related dementia: past, present, and future. J. Alzheimers Dis. 33, 913-922 (2013).

299. De Lepeleire, J., Bouwen, A., De Coninck, L. \& Buntinx, F. Insufficient lighting in nursing homes. J. Am. Med. Dir. Assoc. 8, 314-317 (2007).
300. Figueiro, M. G. et al. Tailored lighting intervention improves measures of sleep, depression, and agitation in persons with Alzheimer's disease and related dementia living in long-term care facilities. Clin. Interv. Aging 9, 1527-1537 (2014)

301. Riemersma-van der Lek, R. F. et al. Effect of bright light and melatonin on cognitive and noncognitive function in elderly residents of group care facilities: a randomized controlled trial. JAMA 299, 2642-2655 (2008).

This double-blinded randomized controlled trial of 189 elderly residents of a group care facility demonstrates that a combination of melatonin supplementation and scheduled light exposure improves cognitive symptoms of dementia and reduces aggression.

302. Singer, C. et al. A multicenter, placebo-controlled trial of melatonin for sleep disturbance in Alzheimer's disease. Sleep 26, 893-901 (2003).

303. Coogan, A. N. et al. The circadian system in Alzheimer's disease: disturbances, mechanisms, and opportunities. Biol. Psychiatry 74, 333-339 (2013).

304. Arey, R. N. et al. An important role for cholecystokinin, a CLOCK target gene, in the development and treatment of manic-like behaviors. Mol. Psychiatry 19, 342-350 (2014)

305. Coque, L. et al. Specific role of VTA dopamine neuronal firing rates and morphology in the reversal of anxiety-related, but not depression-related behavior in the Clocks 19 mouse model of mania. Neuropsychopharmacology 36, 1478-1488 (2011).

306. Dzirasa, K. et al. Lithium ameliorates nucleus accumbens phase-signaling dysfunction in a genetic mouse model of mania. J. Neurosci. 30, 16314-16323 (2010).

307. McClung, C. A. Circadian rhythms, the mesolimbic dopaminergic circuit, and drug addiction. ScientificWorldJournal 7, 194-202 (2007).

308. McClung, C. A. et al. Regulation of dopaminergic transmission and cocaine reward by the Clock gene. Proc. Natl Acad. Sci. USA 102, 9377-9381 (2005).

309. Ozburn, A. R. et al. Direct regulation of diurnal Drd3 expression and cocaine reward by NPAS2. Biol. Psychiatry 77, 425-433 (2015)

310. Ozburn, A. R. et al. NPAS2 regulation of anxiety-like behavior and $\mathrm{GABA}_{\mathrm{A}}$ receptors. Front. Mol. Neurosci. 10,360 (2017).

311. Ozburn, A. R., Larson, E. B., Self, D. W. \& McClung, C. A Cocaine self-administration behaviors in Clock 19 mice. Psychopharmacology 223, 169-177 (2012).

312. Parekh, P. K. et al. Altered GluA1 (Gria1) function and accumbal synaptic plasticity in the Clock 19 model of bipolar mania. Biol. Psychiatry. https://doi.org/ 10.1016/j.biopsych.2017.06.022 (2017).

313. Roybal, K. et al. Mania-like behavior induced by disruption of CLOCK. Proc. Natl Acad. Sci. USA 104 6406-6411 (2007).

This study demonstrates mice harbouring a mutation in Clock display a behavioural repertoire similar to human mania and provides evidence for CLOCK specifically in the ventral tegmental area to be important for these behaviours via modulation of dopamine cell firing.

314. Sidor, M. M. et al. Daytime spikes in dopaminergic activity drive rapid mood-cycling in mice. $\mathrm{Mol}$. Psychiatry 20, 1406-1419 (2015).

315. Spencer $\mathrm{S}$ et al. A mutation in CLOCK leads to altered dopamine receptor function. J. Neurochem. 123, 124-134 (2012)

316. Neufeld-Cohen, A. et al. Circadian control of oscillations in mitochondrial rate-limiting enzymes and nutrient utilization by PERIOD proteins. Proc. Natl Acad. Sci. USA 113, E1673-E1682 (2016)

317. O'Neill, J. S. \& Feeney, K. A. Circadian redox and metabolic oscillations in mammalian systems. Antioxid. Redox Signal. 20, 2966-2981 (2014).

318. Marcheva, B. et al. Circadian clocks and metabolism Handb. Exp. Pharmacol. 217, 127-155 (2013).

319. Braun, R. et al. Universal method for robust detection of circadian state from gene expression. Proc. Natl Acad. Sci. USA 115, E9247-E9256 (2018). This study demonstrates the use of a set of computational algorithms called TimeSignature to predict the endogenous phase of an individual based on the particular gene signatures from the blood acquired at a single timepoint.
320. Jones, S. E. et al. Genome-wide association analyses in 128,266 individuals identifies new morningness and sleep duration loci. PLOS Genet. 12, e 1006125 (2016).

321. Lane, J. M. et al. Genome-wide association analysis identifies novel loci for chronotype in 100,420 individuals from the UK Biobank. Nat. Commun. 7 10889 (2016)

322. LeGates, T. A., Fernandez, D. C. \& Hattar, S. Light as a central modulator of circadian rhythms, sleep and affect. Nat. Rev. Neurosci. 15, 443-454 (2014).

323. LeGates, T. A. et al. Aberrant light directly impairs mood and learning through melanopsin-expressing neurons. Nature 491, 594-598 (2012).

324. Fernandez, D. C. et al. Light affects mood and learning through distinct retina-brain pathways. Cell 175, 71-84 (2018).

Using mice, this study demonstrates that light input to the brain reaches neural circuits related to depression and learning through distinct projections from the retina, revealing a potential mechanism by which aberrant light exposure (for example, light at night) affects mood and cognition.

325. Librodo, P., Buckley, M., Luk, M. \& Bisso, A Chronotherapeutic drug delivery. J. Infus. Nurs. 38 S18-S23 (2015)

326. Ruben, M. D. et al. A database of tissue-specific rhythmically expressed human genes has potential applications in circadian medicine. Sci. Transl Med. 10, eaat8806 (2018)

327. Melkani, G. C. \& Panda, S. Time-restricted feeding for prevention and treatment of cardiometabolic disorders. J. Physiol. 595, 3691-3700 (2017)

328. Mindikoglu, A. L., Opekun, A. R., Gagan, S. K. $\&$ Devaraj, S. Impact of time-restricted feeding and dawn-to-sunset fasting on circadian rhythm, obesity, metabolic syndrome, and nonalcoholic fatty liver disease. Gastroenterol. Res. Pract. 2017, 3932491 (2017).

329. Stewart, K. T., McEachron, D. L., Rosenwasser, A. M. $\&$ Adler, N. T. Lithium lengthens circadian period but fails to counteract behavioral helplessness in rats. Biol. Psychiatry 30, 515-518 (1991)

330. McCarthy, M. J. et al. Genetic and clinical factors predict lithium's effects on PER2 gene expression rhythms in cells from bipolar disorder patients. Trans/ Psychiatry 3, e318 (2013).

331. Sprouse, J., Reynolds, L., Braselton, J. \& Schmidt, A. Serotonin-induced phase advances of SCN neuronal firing in vitro: a possible role for $5-\mathrm{HT}_{5 \mathrm{~A}}$ receptors? Synapse 54, 111-118 (2004)

332. Zhang, R., Lahens, N. F., Ballance, H. I., Hughes, M. E. $\&$ Hogenesch, J. B. A circadian gene expression atlas in mammals: implications for biology and medicine. Proc. Natl Acad. Sci. USA 111, 16219-16224 (2014).

333. Dokucu, M. E., Yu, L. \& Taghert, P. H. Lithium- and valproate-induced alterations in circadian locomotor behavior in Drosophila. Neuropsychopharmacology 30, 2216-2224 (2005).

334. Sathyanarayana, A. et al. Sleep quality prediction from wearable data using deep learning. JMIR Mhealth Uhealth 4, e125 (2016).

\section{Acknowledgements}

Figures were initially designed using BioRender software (biorender.io). Funding for the studies discussed from the authors' group was obtained from NARSAD, International Mental Health Research Organization (IMHRO), MH106460, DA039865, DA037636, MH082876 and DA023988 to C.A.M. and from NARSAD, DA038654 and DA041872 to R.W.L.

\section{Author contributions}

Both authors researched data for article, made substantial contributions to the discussion of the content, wrote the article and reviewed or edited the manuscript before submission.

\section{Competing interests}

The authors declare no competing interests.

\section{Publisher's note}

Springer Nature remains neutral with regard to jurisdictional claims in published maps and institutional affiliations.

Reviewer information

Nature Reviews Neuroscience thanks A. Phillips, K. Wulff and the other anonymous reviewer(s) for their contribution to the peer review of this work. 